\title{
Effects of miR-101-3p on goat granulosa cells in vitro and ovarian development in vivo via STC1
}

Xiaopeng $\mathrm{An}^{1 \dagger}$, Haidong Ma ${ }^{1,2 \dagger}$, Yuhan $\mathrm{Liu}^{1 \dagger}$, Fu Li ${ }^{1}$, Yuxuan Song ${ }^{1}$, Guang $\mathrm{Li}^{1}$, Yueyu Bai ${ }^{3^{*}}$ and Binyun Cao ${ }^{1 *}$ (D)

\begin{abstract}
Background: MiRNAs act as pivotal post-transcriptional gene mediators in the regulation of diverse biological processes, including proliferation, development and apoptosis. Our previous study has showed that miR-101-3p is differentially expressed in dairy goat ovaries compared single with multiple litters. The objective of this research was to explore the potential function and molecular mechanism of miR-101-3p via its target STC1 in goat ovarian growth and development.

Results: cDNA libraries were constructed using goat granulosa cells transfected with miR-101-3p mimics and negative control by RNA-sequencing. In total, 142 differentially expressed unigenes (DEGs) were detected between two libraries, including 78 down-regulated and 64 up-regulated genes. GO and KEGG enrichment analysis showed the potential impacts of DEGs on ovarian development. STC1 was singled out from DEGs for further research owing to it regulates reproductive-related processes. In vitro, bioinformatics analysis and 3'-UTR assays confirmed that STC1 was a target of miR101-3p. ELISA was performed to detect the estrogen (E2) and progesterone (P4) levels. CCK8, EdU and flow cytometry assays were performed to detect the proliferation and apoptosis of granulosa cells. Results showed that miR-101-3p regulated STAR, CYP19A1, CYP11A1 and 3B-HSD steroid hormone synthesis-associated genes by STC1 depletion, thus promoted E2 and P4 secretions. MiR-101-3p also affected the key protein PI3K, PTEN, AKT and mTOR in PI3K-AKT pathway by STC1, thereby suppressing proliferation and promoting apoptosis of granulosa cells. In vivo, the distribution and expression levels of miR-101-3p in mouse ovaries were determined through fluorescence in situ hybridisation (FISH). Immunohistochemistry results showed that STC1 expression was suppressed in mouse ovaries in miR-101-3p-agonist and siRNA-STC1 groups. Small and stunted ovarian fragments, decreased numbers of follicles at diverse stages were observed using Hematoxylin-eosin (HE) staining, thereby showing unusual ovarian development after miR-101-3p overexpression or STC1 depletion. Inhibition of miR-101-3p manifested opposite results.
\end{abstract}

Conclusions: Taken together, our results demonstrated a regulatory mechanism of miR-101-3p via STC1 in goat granulosa cells, and offered the first in vivo example of miR-101-3p and STC1 functions required for ovarian development.

Keywords: Granulosa cells, MiR-101-3p, Ovary, STC1, Transcriptome

\footnotetext{
*Correspondence: baiyueyu_56@163.com; caobinyun@126.com

${ }^{+}$Xiaopeng An, Haidong Ma and Yuhan Liu contributed equally to this work.

${ }^{3}$ Henan Animal Health Supervision Institution, No. 91 Jingsan Road,

Zhengzhou, Henan 450008, P.R. China

${ }^{1}$ College of Animal Science and Technology, Northwest A\&F University, No.

22 Xinong Road, Yangling, Shaanxi 712100, P.R. China

Full list of author information is available at the end of the article
}

(C) The Author(s). 2020 Open Access This article is licensed under a Creative Commons Attribution 4.0 International License, which permits use, sharing, adaptation, distribution and reproduction in any medium or format, as long as you give appropriate credit to the original author(s) and the source, provide a link to the Creative Commons licence, and indicate if changes were made. The images or other third party material in this article are included in the article's Creative Commons licence, unless indicated otherwise in a credit line to the material. If material is not included in the article's Creative Commons licence and your intended use is not permitted by statutory regulation or exceeds the permitted use, you will need to obtain permission directly from the copyright holder. To view a copy of this licence, visit http://creativecommons.org/licenses/by/4.0/ The Creative Commons Public Domain Dedication waiver (http://creativecommons.org/publicdomain/zero/1.0/) applies to the data made available in this article, unless otherwise stated in a credit line to the data. 


\section{Introduction}

In mammals, folliculogenesis and oogenesis proceed in parallel within the ovary [1]. Ovarian follicle consists of an oocyte which surrounded by theca and granulosa cells, and is closely related to ovulation, fertilization, implantation and embryo growth [2]. Granulosa cells preserve and breed oocytes as well as secrete steroid hormones such as estrogen and progesterone, furnishing a crucial microenvironment for follicular growth [3]. Proliferation and differentiation of granulosa cells is elementary for development of follicle and oocyte, ovulation and luteinization, while apoptotic cell death performs the mechanism of ovarian follicle atresia. Thus granulosa cells are supposed to maintain ovarian function $[2,3]$.

MiRNAs are a series of conserved noncoding RNA molecules with 18-25 nucleotides (nts) in length [4]. They can elicit mRNA degradation or translational suppression by pairing with the 3 '-untranslated region (UTR) of their target genes. Subsequently miRNAs reduce gene expression at the post-transcriptional level and undergo a series of physiological events including development, growth, proliferation and apoptosis $[5,6]$. MiRNAs also have vital functions on regulating ovarian growth and development. For example, miR-224 [7], miR-383 [8] and miR-378 [9] regulate steroid hormones secretions in granulosa cells, while miR-145 [10], miR23a [11] and miR-26b [12] take effects on proliferation and apoptosis of granulosa cells by binding to target genes. MiR-21 [13], miR-503 [14], miR-125b and miR145 [15] participate in ovulation, follicular-luteal transformation and luteal process. Based on our prevenient work, we selected miR-101-3p for further research owing to its differentially expression in dairy goat ovaries between single and multiple litter sizes (not published). It's reported that miR-101-3p is a tumour eliminator and implicates a range of tumour-related biological processes. For example, miR-101 suppresses cell proliferation and facilitates cell apoptosis by inhibiting $m T O R$ in Saos-2 cells [16], promotes Bcl2-regulated apoptosis by RLIP76 in prostate cancer cells [17], represses tumour growth and migration by ROCK1 in osteosarcoma cells [18]. However, the functions of miR-101-3p on goat ovaries remain comparatively uncharacterised.

Bioinformatics analysis finds that STC1 is a potential target of miR-101-3p. STC1 is a member of stanniocalcin (STC) family, and the other closely pertinent orthologue is STC2 [19]. STC, a glycoprotein hormone identified in bony fish, can modulate calcium and phosphate levels which produced in the corpuscles of Stannius [20]. In several mammalian tissues, STC1 and STC2 emerge as paracrine/autocrine rather than endocrine compared with their topical glandular expression in fish, thus modulating mineral metabolism [21]. STC1 regulates abundant vital biological processes such as cellular activities, lactation, pregnancy and organogenesis. For example, the elevated expression of STC1 is discovered in breast carcinomas and ovarian cancer, which means STC1 may act as a carcinogenesis factor [22]. The activation of STC1 is observed during gestation and lactation in mouse ovaries, suggesting a gestational and nursing state function [23]. The role of STC1 in ovaries is also enhanced by identifying the subcellular luteal cell targets, cholesterol or lipid storage droplets (steroidogenic active regions) [24]. STC1 shows inhibitory effects on FSH-, LH- and hCG-stimulated progesterone synthesis in rat granulosa cells and bovine luteal cells $[25,26]$. Whereas, it remains indistinct whether STC1 is capable of taking effects on goat ovaries.

In present study, we have accomplished the global transcriptional analysis of miR-101-3p overexpressed goat granulosa cells and identified the DEGs by RNAseqencing (RNA-Seq) method. From the down-regulated DEGs we selected STC1, also a potential target of miR101-3p for further studies on account of its involvement in ovarian function. Then we evaluated the regulatory roles and molecular mechanism of miR-101-3p on steroid hormone synthesis, cell proliferation and apoptosis via targeting STC1 in goat granulosa cells in vitro. Finally we detected the effects of miR-101-3p and STC1 on mouse ovaries in vivo. The current study used in vitro and in vivo models to find out how miR-101-3p and STC1 function on ovarian development.

\section{Materials and methods \\ Cell culture}

The Xinong Saanen dairy goats (1-3 years old, not estrus) in the experimental farm of Northwest A\&F University of China were used. The collected ovaries were washed and maintained in PBS with penicillin $(100 \mu \mathrm{g} / \mathrm{mL})$ and streptomycin $(100 \mu \mathrm{g} / \mathrm{mL})$ and then transferred to culture dishes. Goat granulosa cells were released into the medium when the large antral follicles were punctured by hypodermic needles. HEK293T cells were bought from Shanghai Tongwei Company and thawed from liquid nitrogen directly in $37^{\circ} \mathrm{C}$ sterile water. Granulosa cells or HEK293T cells were cultivated in DMEM/F12 medium (Gibco, Grand Island, USA) or DMEM (high glucose) medium (Gibco, Grand Island, USA) both supplemented with $10 \%$ foetal bovine serum (FBS), penicillin $(100 \mu \mathrm{g} /$ $\mathrm{mL})$ and streptomycin $(100 \mu \mathrm{g} / \mathrm{mL})$ in a humidified atmosphere with $5 \% \mathrm{CO}_{2}$ at $37^{\circ} \mathrm{C}$.

\section{PCDNA3.1-STC1 plasmid construction}

The CDS regions of STC1 (XM_005684015) were extended using PCR derived from the extracted cDNA of goat granulosa cells. The PCR products were digested and cloned into pMD ${ }^{\mathrm{mm}} 19-\mathrm{T}$ vector (TakaRa, Ostu, Japan). Afterwards, STC1 overexpression plasmids were 
constructed using the eukaryotic expression pcDNA3.1(+) vector (Thermo Fisher, Shanghai, China) between Hind III and Xho I sites. The entire STC1 CDS sequences were introduced into the numerous cloning spots of the pcDNA3.1 vector, and the constructs were confirmed through DNA sequencing. The forward and reverse primers of STC1 were Hind III 5'-CCCAAGCTTAGCAA CTTAGCGGAAACT-3' and Xho I 5'-CCGCTCGAGG CGTAAACACCCTTAAAAC-3', respectively.

\section{Transfection and RNA extraction}

Granulosa cells $\left(5 \times 10^{6}\right.$ cells/well $)$ were precultured in 6-well plates. The miR-101-3p mimics (miR-101-3p-mi), mimics negative control (NC-mi), miR-101-3p inhibitors (miR-101-3p-in), inhibitors NC (NC-in), siRNA-STC1 (si-STC1), siRNA negative control (NC) (GenePharma, Shanghai, China), pcDNA3.1 and pcDNA3.1-STC1 vectors were transfected into granulosa cells by Lipofectamine 2000 (Invitrogen, Carlsbad, USA). Table S1 shows the sequences. Optimal medium concentration of mimics or inhibitors were used in this study based on the manufacturer's standards. Briefly, $5 \mu \mathrm{L}$ miR-101-3p mimics/NC-mi $(50 \mathrm{nmol} / \mathrm{L})$ or inhibitors/NC-in (100 $\mathrm{nmol} / \mathrm{L})$ or si-STC1/NC $(50 \mathrm{nmol} / \mathrm{L})$ with $5 \mu \mathrm{L}$ Lipofectamine 2000, $5 \mu \mathrm{g}$ pcDNA3.1-STC1/pcDNA3.1 vectors with $6 \mu \mathrm{L}$ Lipofectamine 2000, $2.5 \mu \mathrm{L}$ miR-101-3p-mi and $2.5 \mu \mathrm{g}$ pcDNA3.1-STC1 vectors with $6 \mu \mathrm{L}$ Lipofectamine 2000 were diluted in $200 \mu \mathrm{L}$ Opti-MEM I medium (Gibco, CA, USA) and cultivated for $20 \mathrm{~min}$. Then the medium was replaced within 4-6h. After 24 or $48 \mathrm{~h}$ transfection, the cells were harvested for further detection. RNA was extracted and purified by TRIzol reagent (Invitrogen, CA, USA). Agilent 2100 Bioanalyzer (Agilent Technologies, CA, USA) was used to evaluate RNA concentration and purity. The ratio of the optical densities estimated at 260 and $280 \mathrm{~nm}$ was $>1.8$ and $<2.0$ for all RNA samples.

\section{Library preparation for sequencing}

After goat granulosa cells transfected with miR-101-3p mimics and negative control (NC) for $24 \mathrm{~h}$ (each treatment had three repeats), the extracted RNA were used to construct RNA libraries. The optical densities of 260/ $280 \mathrm{~nm}$ in miR-101-3p mimics group were 1.91, 1.95 and 1.90, those of NC group were 1.88, 1.94 and 1.80 . Library preparation for sequencing of each experimental sample was instituted based on the manufacturer's standards. In brief, mRNA was enriched using oligo magnetic beads after total RNA extraction. The purified mRNA was first divided to $200-300 \mathrm{bp}$ fragments with an RNA fragmentation kit. Utilising random hexamer primers and reverse transcriptase, the first-strand cDNA synthesis was accomplished. Afterwards, a tailored second-strand primer and strand synthesis enzyme mix were added, followed by incubation with dNTPs, DNA polymerase I and RNase $\mathrm{H}$ to synthesise the second strand. The mixes were subjected to end repair with an End Prep enzyme mix after purification using an Agilent 2100 bioanalyzer, supplemented with adaptor ligation, single A base and agarose gel insulation of 300-400 bp cDNA. The library outcomes were used for sequencing reaction in an Illumina HiSeq 2000 platform.

\section{Read mapping on the goat reference genome and data analysis}

The information was converted into sequence data, and the premier image was selected. After the 3 'adaptor sequences were filtered, low-quality reads (the percentage of low quality measured with $Q$ value of $\leq 5$ was more than $50 \%$ in each read) with more than five Ns per 100 bp and superfluous reads were separated. The clean reads were arranged according to the goat reference genome (GCF_001704415.1_ARS2 _genomic.fa) for convergence by Top Hat v2.0.12.

The correlation among the repeated biological samples was checked to ensure that the selection of samples was reasonable. The square $\left(\mathrm{R}^{2}\right)$ of Pearson correlation coefficient was used to represent the similarity of expression patterns between samples. $R^{2}>0.92$ was considered as the ideal sampling and experimental condition. All the spliced forms of the transcript were determined based on Stringtie, and new transcript regions were found using gffcompare compared with the reference genome. According to the genetic model predicted by Stringtie for each sample, ASprofile software (http://ccb.jhu.edu/software/ASprofile/) was used to classify and count the variable splicing events. InDel sites were obtained by Varscan (version 2.3.7).

\section{Standardised expression levels of genes and screening of DEGs}

Reads per kilobase transcriptome per million mapped reads $(R P K M)(R P K M=$ entire exon reads/mapped reads in millions $\times$ exon length in $\mathrm{kb}$ ) method was used to normalise the gene expression levels. RPKM $>1$ was used as the threshold to judge gene expression. Differentially expressed unigenes (DEGs) and their comparative $P$-values were calculated according to previous study [27]. The significance limitation of the $P$-value in numerous tests was fixed on the basis of FDR. Standardised gene expression levels of groups were measured using the fold changes $\left(\log _{2} \mid\right.$ Ratio $\left.\mid\right)$ by DESeq (version 1.18.0). Finally, the standards of (i) $P$-value $<0.05$ and (ii) $\log _{2}$ $\mid$ Ratio $\mid>1$ were utilised to determine the significance of gene expression differences. Volcano and MA plot maps of DEGs were created using the $\mathrm{R}$ language ggplots2 software package. 


\section{Gene Ontology and KEGG pathway analysis of DEGs}

The DEGs were categorised into molecular function, cellular component and biological process using Gene Ontology (GO) annotation [28]. Hypergeometric detection was implemented to match all DEGs to terms in the database (http://www.geneontology.org/) and to examine the apparently enriched GO terms of DEGs. Next, we used KEGG (http://www.genome.jp/kegg/), an elementary public pathway-correlative database to appraise remarkably enriched signal transduction pathways or metabolic pathways of DEGs [29]. The valuation formula was the same as the value of GO annotation.

\section{Quantitative real-time PCR (RT-qPCR)}

For the mRNA expression level, $0.3 \mathrm{mg}$ of total RNA was synthesized into cDNA using PrimeScripts RT Reagent Kit (TakaRa, Ostu, Japan) according to the manufacturer's specifications. After reverse transcription, TB Green ${ }^{\text {tw }}$ Premix Ex Taq ${ }^{\text {Tax }}$ II (TakaRa, Ostu, Japan) was used to quantify the relative amount of miR-101-3p and disparate genes by the CFX Connect Real-Time PCR Detection System (BioRad, Hercules, USA). Table S2 shows the validated primers used for RT-qPCR. In brief, the amplification reaction mixtures of $25 \mu \mathrm{L}$ contained $2 \mu \mathrm{L}$ cDNA, $12.5 \mu \mathrm{L}$ SYBR Premix Ex Taq II, $1 \mu \mathrm{L}$ PCR Forward and Reverse

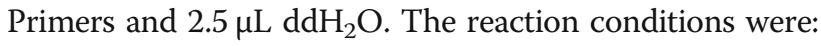
initial denaturation for $30 \mathrm{~s}$ at $95^{\circ} \mathrm{C}$, denaturation for $5 \mathrm{~s}$ at $95^{\circ} \mathrm{C}$, followed by 40 cycles of annealing for $30 \mathrm{~s}$ at $60^{\circ} \mathrm{C}$ and extending for $50 \mathrm{~s}$ at $72^{\circ} \mathrm{C}$. At the end of the total runs, a melting-curve analysis $\left(95^{\circ} \mathrm{C}\right.$ for $15 \mathrm{~s}$ and $60^{\circ} \mathrm{C}$ for $1 \mathrm{~min}$ at $0.5^{\circ} \mathrm{C} / 5 \mathrm{~s}$ until $95^{\circ} \mathrm{C}$ ) was performed to ensure the specificity of amplification. U6 or $\beta$-actin was detected as a loading control for miRNA or genes mRNA levels. Each condition was repeated in three wells, all experiments were performed in triplicate. Relative expression was calculated through the $2^{-\Delta \Delta \mathrm{Ct}}$ approach.

\section{Western blot}

After each transfection for $48 \mathrm{~h}$, granulosa cells were collected and lysed with the ice-cold RIPA lysis buffer (Bioteke, Beijing, China) supplemented with $0.1 \mathrm{mg} / \mathrm{mL}$ PMSF (Solarbio, Beijing, China). The mixtures were centrifuged at $4{ }^{\circ} \mathrm{C}$ and proteins were then extracted from the cells. BCA Protein Assay kit (Vazyme Biotech, Nanjing, China) determined the protein concentration. SDS-PAGE (12\%) was performed with $40 \mu \mathrm{g}$ total protein approximately via electrophoresis, after which the proteins were diverted into polyvinylidene difluoride membranes (Merck Millipore, Darmstadt, Germany). After shaking in 5\% non-fat dry milk at room temperature for $2 \mathrm{~h}$, the membranes were incubated with primary antibodies at $4{ }^{\circ} \mathrm{C}$ overnight and species-specific secondary antibodies for $2 \mathrm{~h}$, which were eventually colour-analysed with a Beyo ECL Star kit (Beyotime, Shanghai, China). The primary antibodies were as follows: HSD3B1 (1:1000, Abcam, London, UK), STAR (1:1000, Abcam, London, UK), CYP11A1 (1:600, Bioss, Beijing, China), TP53 (1:500, BBI, Shanghai, China), CASP3 (1:700, BBI, Shanghai, China), ACTB (1:1000, BBI, Shanghai, China), STC1 (1:700, ABclonal, Wuhan, China), PI3K (1:400, ABclonal, Wuhan, China), mTOR (1:600, ABclonal, Wuhan, China), p-mTOR (1:600, ABclonal, Wuhan, China), AKT (1:600, ABclonal, Wuhan, China), p-AKT (1:400, ABclonal, Wuhan, China), PTEN (1:900, ABclonal, Wuhan, China), Bcl-2 (1:500, ABclonal, Wuhan, China), BAX (1:500, ABclonal, Wuhan, China), CCND1 (1:800, ABclonal, Wuhan, China), CDK4 (1:600, ABclonal, Wuhan, China), CCNE1 (1:600, ABclonal, Wuhan, China) and PCNA (1:500, ABclonal, Wuhan, China). The transfection efficiency of miR-101-3p and STC1 is showed in Fig. S1.

\section{3'-UTR luciferase reporter assay}

The sequences of STC1 3'-UTR containing the predicted target sites of miR-101-3p were amplified from goat genomic DNA through PCR. Afterwards, the PCR fragments were inlet into psiCHECK-2 vectors with particular Xhol I and Not I restriction enzymes for dualluciferase assay. Forward primer of wild-type (wt) STC1: Xho I 5'-CCGCTCGAGTAAGGTCTAACTGGAATA3', reverse primer: Not I 5'-AAATATGCGGCCGCAT CTCATACAGGCCCAT-3'. To institute mutated 3'UTR reporter vectors, the calculated binding sites were mutated by site-directed mutagenesis with specific primers. Forward primer of mutant (mu)-type STC1: Xho I 5'-GGCTCGAGTCCTCAGTGTCTAATTTCCT3', reverse primer: Not I 5'-TAGCGGCCGCCTTTGT TTAAGCAGAGTCCT-3'. HEK293T cells were seeded in a 48-well plate and then co-transfected $10 \mathrm{pmol}$ miR101-3p-mi/NC-mi or miR-101-3p-in/NC-in with 0.33 $\mathrm{mg} \mathrm{wt}-/ \mathrm{mu}$ - luciferase reporter gene plasmids using $\mathrm{Li}$ pofectamine 2000 for $48 \mathrm{~h}(n=3)$. The renilla and firefly luciferase activities were evaluated using a Dual-Glo luciferase assay system (Promega, Madison, USA) with Thermo Scientific Varioskan Flash (Thermo Scientific, Shanghai, China). Firefly luciferase activity was normalized to renilla luciferase activity.

\section{ELISA}

Granulosa cells were seeded and harvested in 6-well plates when post-transfected in $24 \mathrm{~h}$. Cell-free supernatants were assembled and used in the evaluation of oestrogen (E2) and progesterone (P4) production with an enzyme-linked immunosorbent assay (ELISA) kit (Zhenke, Shanghai, China). Based on the kit specifications, the absorbance at $450 \mathrm{~nm}$ with $50 \mu \mathrm{L}$ supernatants was set using an Epoch microplate reader (Biotek, Winooski, USA). The corresponding concentrations of the samples were calculated using the Equation from the 
linear regression of the standard curve was obtained and then calculated corresponding concentrations of the samples. The mean intra- and inter-assay variable coefficient values were less than $15 \%$, and the sensitivity of kits was $1 \mathrm{pmol} / \mathrm{mL}$.

\section{CCK8 assay}

The viability of granulosa cells was examined via CCK8 assay. Granulosa cells were transfected and cultured in a $100 \mu \mathrm{L}$ volume in 96 -well plates $\left(1 \times 10^{4}\right.$ cells/well $)$ with six repetitions and then treated in gradient times (12, 24, 36 and $48 \mathrm{~h}$ ). Then each well was added $10 \mu \mathrm{L}$ CCK 8 reagents (ZETATM life, Beijing, China) and cultivated for $3 \mathrm{~h}$ at $37^{\circ} \mathrm{C}$ in the dark. After 20 min of shaking at room temperature, Epoch microplate reader (Biotek, Winooski, USA) was used to detect the absorbance of mixtures at $450 \mathrm{~nm}$.

\section{EdU staining}

The proliferation of granulosa cells was examined via EdU staining. Granulosa cells were transfected and cultured in a $100 \mu \mathrm{L}$ volume in 96-well plates $\left(1 \times 10^{4}\right.$ cells/ well) with three repetitions and harvested after transfection for $24 \mathrm{~h}$. Then cells were stained with EdU (Ribobio, Guangzhou, China) for $2 \mathrm{~h}$ at a final concentration of $50 \mu \mathrm{mol} / \mathrm{L}$ and with DAPI at room temperature for 15 min after PBS washed three times. The cells were observed by Fluorescence microscopy (IX71, Olympus, Japan).

\section{Flow cytometry assay (FCM)}

Granulosa cells were seeded and harvested in 6-well plates when post-transfected in $24 \mathrm{~h}$. Then the cell cycle of granulosa cells was measured using cell cycle staining kit (SeaBiotech, Shanghai, China) according to the manufacturer's specifications. The apoptosis of granulosa cells were measured using Annexin V-FITC PI staining apoptosis assay kit (SeaBiotech, Shanghai, China), and the percentage of apoptotic cells were detected behind $200 \mu \mathrm{L}$ mixtures staining with $10 \mu \mathrm{L}$ annexin V-FITC and $5 \mu \mathrm{L}$ propidium iodide (PI) in $30 \mathrm{~min}$. All the flow cytometry assays were detected with a FACSCalibur flow cytometer (BD Biosciences).

\section{Animal experimental treatment}

Twelve healthy SPF C57BL/6j female mice aged 7-8 weeks and weighing $22 \pm 2 \mathrm{~g}$ were selected. The mice were provided by Xi'an Jiaotong University Medical Laboratory Animal Center (approval number: SCXK (Shaanxi) 2007-001). All animals were under protocols approved by the Institutional Animal Care and Use Committee. After 1 week of adaptive feeding, the mice were randomly divided into four groups and injected with intraperitoneal drugs on the $1^{\text {st }}, 3^{\text {rd }}$ and $7^{\text {th }}$ day.
The test control group was named NC $(100 \mu \mathrm{L}$ saline), whereas the test groups were named miR-101$3 \mathrm{p}$ agonist (miR-101-3p-ag; $10 \mathrm{nmol}$ per time, with $100 \mu \mathrm{L}$ saline), miR-101-3p antagonist (miR-101-3pantag; $20 \mathrm{nmol}$ per time, with $100 \mu \mathrm{L}$ saline) or siRNA-STC1 (si-STC1; $20 \mathrm{nmol}$ per time, with $100 \mu \mathrm{L}$ saline) (RiboBio, Guangzhou, China). On the $9^{\text {th }}$ day, mice ovaries were separated and collected under sterile conditions and then fixed with $4 \%$ paraformaldehyde after washed with pre-cooled normal saline [30].

\section{Fluorescence in situ hybridisation (FISH)}

After tissue fixation, dehydration, slicing and dewaxing paraffin sections to water, the sections were boiled in the repair solution for $10 \mathrm{~min}$. Proteinase $\mathrm{K}(20 \mu \mathrm{g} / \mathrm{mL})$ was added dropwise and digested at $37^{\circ} \mathrm{C}$ for $30 \mathrm{~min}$ and wash with PBS for $5 \mathrm{~min}$ at 3 times. Then the prehybridisation solution was added and incubated at $37^{\circ} \mathrm{C}$ for $1 \mathrm{~h}$. The mmu-miR-101a probe containing a hybridisation solution was added at a concentration of $8 \mathrm{ng} / \mu \mathrm{L}$, and hybridisation was performed at $37^{\circ} \mathrm{C}$ overnight. A DAPI staining solution was added to the sections and incubated for $8 \mathrm{~min}$ in the dark, and anti-fluorescence quenching sealing tablets were mounted after washing. The nucleus stained by DAPI (Servicebio, Wuhan, China) was blue under ultraviolet excitation, and the positive expression was green fluorescence of the corresponding fluorescein-labelled FAM (488). The images were observed using a upright fluorescence microscope (Nikon, Tokyo, Japan).

\section{Immunohistochemistry}

Control and treated ovaries were collected and fixed in $4 \%$ paraformaldehyde, incubated in a $20 \%$ sucrose solution at $4{ }^{\circ} \mathrm{C}$ overnight. Then the sections were added with $0.3 \%$ hydrogen peroxide in methanol solution and permeabilised with $0.3 \%$ Triton X100 for 30 $\mathrm{min}$. The sections were rinsed three times $(5 \mathrm{~min}$ each) in PBS at room temperature. Serum dilution $(100 \mathrm{~mL}$ PBS with $1 \mathrm{~g}$ bovine serum albumin and $0.08 \mathrm{~g}$ sodium azide) was applied to STC1 primary antibodies (1:300, Abcam, London, UK) at $4{ }^{\circ} \mathrm{C}$ overnight. After washed in PBS for $5 \mathrm{~min}$ in three times, a biotin-conjugated anti-rabbit secondary antibody (1: 100, Invitrogen, Carlsbad, USA) was added at room temperature for $2 \mathrm{~h}$. Finally, the sections were incubated with SABC-DyLight 488 (SABC, 1:800, Boster, Shanghai, China) and DAPI (1:1000, Boster, Shanghai, China) for $30 \mathrm{~min}$. SABC-positive cells were dyed in brown, while cell nuclei were dyed with DAPI in blue. Image Pro Plus image analysis software was used for quantitative immunohistochemical analysis. 


\section{Hematoxylin-eosin (HE) staining}

The dissected ovaries were fixed in $4 \%$ paraformaldehyde and either frozen in cryomatrix or embedded in paraffin. The sections were stained with Harris hematoxylin (Bioss, Beijing, China) for $7 \mathrm{~min}$, washed with tap water and stained in eosin staining solution (Bioss, Beijing, China) for 1-3 min. The sections were treated with neutral balsam after drying, and observed by fluorescence microscope (Nikon, Tokyo, Japan).

\section{Statistical analysis}

All data were analyzed by SPSS 19.0 and presented as mean \pm SD of three independent experiments. Student's ttest or one-way ANOVA followed by determination of the least significant difference (LSD) for post-hoc multiple comparisons was applied to compare differences of means between two or among more than two groups using GraphPad Prism 7 software. Significance levels or $P$ values were stated in each corresponding figure legend. Significance was accepted at the level of $P<0.05$ $\left({ }^{*} P<0.05,{ }^{* *} P<0.01\right)$.

\section{Results}

\section{Molecular analysis and aligning to sequencing data}

To obtain a global scope of sequencing transcriptome, total RNA from goat granulosa cells transfected with miR-101-3p mimics and NC was used to institute RNA libraries through RNA-Seq. Prior to high throughput sequencing, molecular analysis was performed to evaluate the miR-101-3p expression levels. We acquired 72,795, 015 and 73,658,977 clean reads per sample after filtering the unique adaptor sequences consisting of $\mathrm{N}$ and lowquality sequences. Approximately $85.313 \%$ and $85.307 \%$ total mapped reads were acquired from the reference genome (Table 1). In the miR-101-3p group, $3.612 \%$ or $81.656 \%$ was mapped either to multiple or to unique genomic locations, whereas the $\mathrm{NC}$ group exhibited $3.135 \%$ or $82.171 \%$ reads. The distribution of effective sequences in the reference genomes was measured using the standard metrics of exon, intron and intergenic reads. Table S2 shows the individual distributions. Before performing differential expression analysis, the correlation among the repeated samples was examined. The heat map exhibited that $R^{2}$ values among three replicates originating from the same treatment were greater than 0.95 (Fig. S2a), showing the slight differences among samples in each treatment.

\section{Analysis of DEGs after miR-101-3p overexpression}

Next we examined the differentially expressed unigenes after miR-101-3p overexpression to determine the underlying mechanisms mediated by miR-101-3p. As multiple DEGs were present between two libraries, genes coinciding with the specified criterion of $\mid \log _{2}$ FoldChange $\mid>1$ and $P$-values $<0.05$ were centralised. We identified 142 DEGs compared miR101-3p with NC libraries, including 78 down-regulated and 64 up-regulated genes (Fig. S2b). Noticeably, in the miR-1013p group, the five down-regulated DEGs that expressed most significant were NDUFA4L2 $(-1.24769817$-fold, $P=8.87 \mathrm{E}$ 50), C4BPA (-1.490250616-fold, $P=3.95 \mathrm{E}-29)$, STC1 $\quad$ 1.160429719-fold, $\quad P=4.25 \mathrm{E}-27), \quad$ LOC102189835 (1.033859332-fold, $P=1.10 \mathrm{E}-12)$ and MCOLN3 (1.474046641-fold, $P=1.05 \mathrm{E}-5)$ as well as up-regulated DEGs were FSHB (1.358101058-fold, $P=4.42 \mathrm{E}-13), \quad B M P E R$ (1.375372372-fold, $P=3.28 \mathrm{E}-6)$, ADAMTS15 (2.00772808fold, $P=9.94 \mathrm{E}-6), \quad$ LOC102185049 (1.101564295-fold, $P=$ 2.32E-5) and LOC102178901 (2.699102983-fold, $P=3.59 \mathrm{E}-5$ ) compared with NC. The five down-regulated DEGs with highest fold change were LOC108633912 (inf-fold, $P=$ $0.001479515), \quad L O C 102187646$ (inf-fold, $P=0.031226999$ ), C3H1orf168 (inf-fold, $P=0.023013464), E D N 2$ (- 4.13414302fold, $P=0.035939657$ ) and RETN (-3.545297992-fold, $P=$ $0.021438006)$ as well as the five up-regulated DEGs were FAM159A (inf-fold, $P=0.020739771$ ), KRT14 (inf-fold, $P=$ 0.012713064 ), LOC106501921 (inf-fold, $P=0.023789854$ ), SEZ6 (inf-fold, $P=0.024290615$ ) and $K N D C 1$ (inf-fold, $P=$ 0.03442371). All up- and down-regulated DEGs identified are presented in Table S4.

\section{Functional classification analysis of DEGs}

To further explore the physiological processes associated with DEGs, we implemented GO analysis by operating queries for the respective DEG against the GO database, which furnishes information correlated with three independent ontology categories: cellular component, molecular function and biological process [28]. Fig. S2c and

Table 1 Summary of sequence read alignments to the reference genome

\begin{tabular}{lllll}
\hline Category & miR-101-3p & & Negative control (NC) \\
\cline { 2 - 3 } & Reads number & Percentage & & Reads number \\
\hline Total reads & $72,795,015$ & $100 \%$ & $73,658,977$ & Percentage \\
Total mapped reads & $62,103,473$ & $85.313 \%$ & $62,835,877$ & $100 \%$ \\
Multiple mapped reads & $2,660,677$ & $3.655 \%$ & $2,309,196$ & $85.307 \%$ \\
Uniquely mapped reads & $59,442,796$ & $81.656 \%$ & $60,526,526$ & $3.135 \%$ \\
\hline
\end{tabular}

Total reads: total number of sequencing reads. Total mapped reads: the reads that can aligned to reference sequence and the ratio of it. Multiple mapped reads: in total mapped reads, reads aligned to two or more places. Uniquely mapped reads: in total mapped reads, reads aligned to only one postion 
Table S5 show the results of the GO analysis of DEGs. Overall, the DEGs were categorised into 712 functional groups and subsequently into three ontologies, that is, 70 (9.8\%), 569 (79.9\%) and $73(10.3 \%)$ terms in accordance with molecular functions, biological processes and cellular components, respectively. Binding (GO:0005488, 23 out of 56 genes), single-multicellular organism process (GO:0044707, 30 out of 56 genes) and cell part (GO:0044464, 39 out of 56 genes) were mostly dominant terms in each primary category.

Diverse genes generally cooperate with one another to perform their biological capabilities. KEGG is an effective pathway-related database and pathway enrichment analysis of enriched metabolic or signal transduction pathways of DEGs [29]. Among those genes with KEGG pathway annotation, about 84.5\% DEGs (120/142) were identified in 68 enriched pathways (Fig. S2d and Table S6). The pathway term that exhibited the highest level of significance was neuroactive ligand-receptor interaction (KO:04080, $P=$ 0.006076663) with seven DEGs. Natural killer cell-mediated cytotoxicity (KO:04650, $P=0.005498505)$ and cytokinecytokine receptor interaction (KO:04060, $P=0.05040407$ ) were also among the significantly enriched pathways with five DEGs each. Complement and coagulation cascades (KO:04610, $P=0.003356877$ ) with four DEGs, natural killer cell-mediated cytotoxicity (KO:04650, $P=0.005498505)$ with five DEGs and neuroactive ligand-receptor interaction (KO:04080, $P=0.006076663$ ) with seven DEGs were the three most notably enriched pathways.

\section{Verification of differential gene expression through RT- qPCR}

We selected 12 down-regulated (STC1, NDUFA4L2, C4BPA, LOC102189835, CLEC9A, MCOLN3, RETN, SLC11A1, C1QC, IRF8, S100A9 and CCDC33) and 8 upregulated (FSHB, KRT7, LOC102185049, CNNM1, GUCY2C, BMPER, ADAMTS15 and LOC102178901) genes to verify the expression profiles acquired through RNASeq. The RT-qPCR results showed that compared with NC, miR-101-3p inhibited STC1, NDUFA4L2, C4BPA, MCOLN3, RETN, SLC11A1, C1QC, S100A9 and CCDC33 as well as promoted FSHB, CNNM1, GUCY2C, BMPER and LOC102178901 in mRNA levels significantly. Although the mRNA differences of LOC102189835, CLEC9A, IRF8, ADAMTS15, KRT7 and LOC102185049 weren't prominent, they were still consistent with the sequencing detections (Fig. 1). The linear regression method proved that the correlation coefficient $R^{2}=0.77$ (Fig. 1). Hence, RNA-Seq can yield appropriate data for mRNA distinct expression analysis after miR-101-3p overexpression in granulosa cells.

\section{MiR-101-3p specifically targets STC1}

Based on a target prediction algorithm (TargetScan, http://www.targetscan.org/), we probed into the novel underlying molecular targets of miR-101-3p. Among these potential targets, STC1 attracted our attention because it was not only a key regulator of diverse metabolic processes [22-26], but also a DEG in terms of RNA-Seq approach. As miRNA functions and suppresses the expression of target genes by binding to 3 'UTR, we implemented a two-tier luciferase assay in HEK293T cells using the psiCHECK-2 vector and framed luciferase reporters with the wt- or mu-STC13'-UTR (Fig. 2a), respectively. Figure $2 \mathrm{~b}$ shows that compared with control, miR-101-3p overexpression prominently inhibited relative luciferase activities in cells transfected with psiCHECK2-wt-STC1 but not in the mutant plasmid. The opposite results were found in miR-101-3p-in-treated cells (Fig. 2b).

To affirm whether STC1 expression is regulated by miR-101-3p, we detected its mRNA and protein levels in granulosa cells using RT-qPCR and Western blot respectively. We observed significant STC1 mRNA decrease in miR-101-3p-mi group and increase in miR101-3p-in group compared with controls (Fig. 2c). The changes in the protein expression of STC1 showed the same results (Fig. 2d), revealing that miR-101-3p targets the 3'-UTR of STC1 specifically and functions as a demotivated mediator.

\section{MiR-101-3p promotes steroid hormone synthesis via STC1} After transfection of miR-101-3p mimics or inhibitors in granulosa cells for $24 \mathrm{~h}$, the levels of E2 and P4 in the cell-free supernatants were detected by ELISA to investigate the effects of miR-101-p on steroid hormone synthesis. Cells with miR-101-3p overexpression showed enhanced E2 and P4 secretions (Fig. 3a). MiR-101-3p inhibition decreased E2 production but had no significant effects on P4 (Fig. 3a). Subsequently, we probed into whether miR-101-3p functions on steroid hormone synthesis of granulosa cells by inhibiting STC1. A vector containing STC1 coding sequence (CDS) or si-STC1 was transfected into granulosa cells for $24 \mathrm{~h}$. STC1 overexpression blocked E2 and P4 secretions, which were improved in si-STC1-treated cells (Fig. 4a). Meanwhile, STC1 significantly reduced E2 and P4 secretions when miR-101-3p was overexpressed (Fig. 4b).

E2 and P4, as vital steroid hormones, their synthesis depends on a series of enzymatic transformation processes [31]. In order to further study the regulation of miR-101$3 p$ in steroid hormones synthesis, RT-qPCR and Western blot were used to detect the expressions of steroid hormone synthesis-associated vital genes (CYP11A1, CYP19A1, 3 $\beta-H S D$ and STAR) in granulosa cells. At mRNA levels, miR-101-3p elevation increased CYP11A1 and $3 \beta-H S D$ expression levels but not CYP19A1 and STAR (Fig. 3b). However, no remarkable effects were observed between miR-101-3p-in and NC-in groups (Fig. 


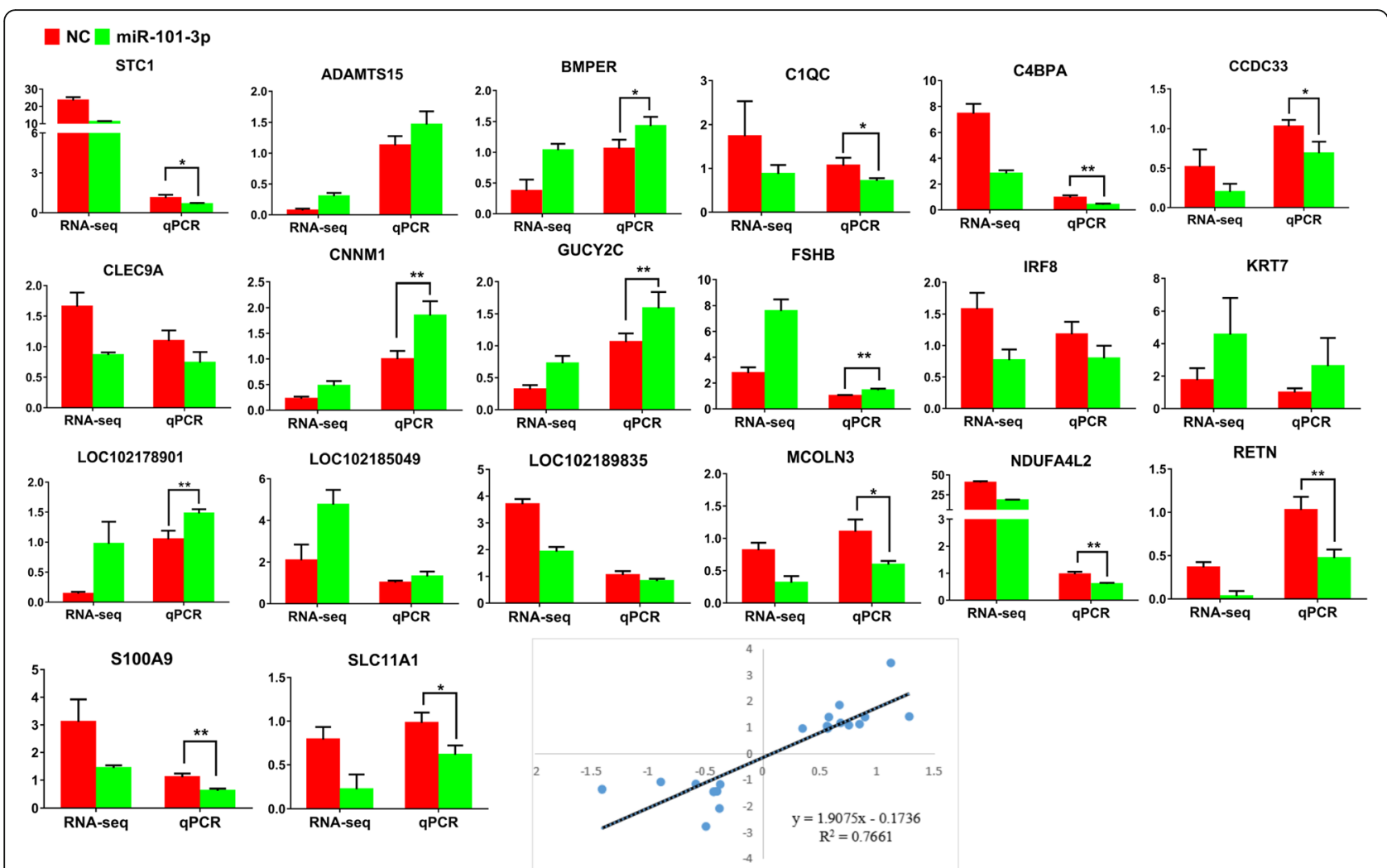

Fig. 1 Verification of differential genes expressions. RT-qPCR quantifies 12 down-regulated DEGs (STC1, NDUFA4L2, C4BPA, LOC102189835, CLEC9A, MCOLN3, RETN, SLC11A1, C1QC, IRF8, S100A9 and CCDC33) and 8 up-regulated DEGs (FSHB, KRT7, LOC102185049, CNNM1, GUCY2C, BMPER, ADAM TS15 and LOC102178901) in goat granulosa cells, which are transfected with miR-101-3p mimics or negative control (NC) for $24 \mathrm{~h}$. $\beta$-actin is used as an internal control. The linear regression equation indicates the correlation coefficient $R^{2}$ value between genes. Values are expressed as mean \pm SD of $n=3 .{ }^{*} P<0.05,{ }^{* *} P<0.01$

3b). At protein levels, CYP19A1, 33-HSD and STAR expressions increased, whereas CYP11A1 expression decreased in miR-101-3p-mi group (Fig. 3c). MiR-101-3p

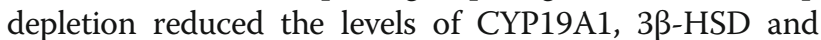
STAR as well as increased CYP11A1 expression significantly (Fig. 3c). Decline of STC1 promoted the protein levels of CYP11A1, CYP19A1, 33-HSD and STAR. On the contrary, suppressive expressions of CYP11A1, CYP19A1 and $3 \beta-\mathrm{HSD}$ in pcDNA3.1-STC1 group were observed (Fig. 4c). Co-expression of miR-101-3p with STC1 exhibited an adiaphorous effect on CYP19A1, 3 $\beta$-HSD and STAR but not on CYP11A1 (Fig. 4d). These findings demonstrate that miR-101-3p promotes E2 and P4 secretions in granulosa cells via $S T C 1$ by regulating CYP11A1, CYP19A1, 3B-HSD and STAR steroid hormone synthesisassociated genes.

\section{MiR-101-3p inhibits granulosa cell proliferation via STC1} CCK8 and EdU analyses were performed for the detection of cell viability and proliferation following cell transfected with miR-101-3p mimics or inhibitors. Granulosa cell viability was standardized by relative absorbance (OD values at $450 \mathrm{~nm})$. The results showed a slight decrease in relative absorbance after miR-101-3p overexpression. By contrast, miR-101-3p inhibition significantly increased cell viability compared with control in 12, 24 and $48 \mathrm{~h}$ (Fig. 5a). These results were similar with those of the EdU staining assay, showing decrease or increase in the number of Sphage cells after treatment with miR-101-3p mimics or inhibitors (Fig. 5b). The influence of miR-101-3p on granulosa cell cycle was detected using flow cytometry. Granulosa cells after miR-101-3p overexpression showed 93.11\% cells at G0/G1 phase, $3.43 \%$ cells at $\mathrm{S}$ phase and $3.46 \%$ cells at G2 phase compared to $83.15 \%, 6.89 \%$ and $6.96 \%$ cells in NC group (Fig. 5b). We observed reductive or incremental cell numbers at G0/G1 (88.51\%) or S phase (7.88\%), but with no prominent effects on cells at G2 phase $(3.61 \%)$ in miR-101-3p-in group compared with the percentages in NC-in group (93.41\%, 3.32\% and 3.27\%) (Fig. 5b). Thus, we conclude that miR-101-3p inhibits the proliferation of goat granulosa cells. After treatment in 12, 24, 36 and $48 \mathrm{~h}$, granulosa cell viability was notably inhibited in si-STC1 group compared with NC. The cell viability was elevated in $24 \mathrm{~h}$ between pcDNA3.1-STC1 and control groups (Fig. 5a). Cells transfected with si-STC1 or pcDNA3.1-STC1 vectors decreased or increased the 

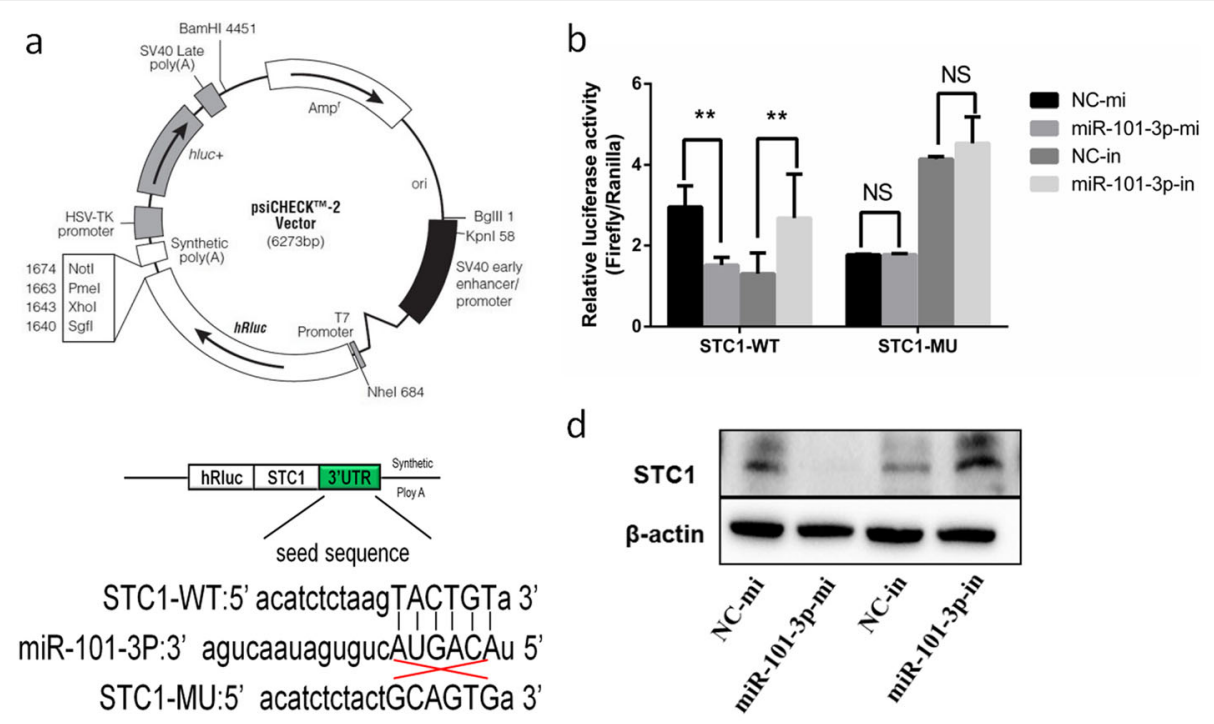

d
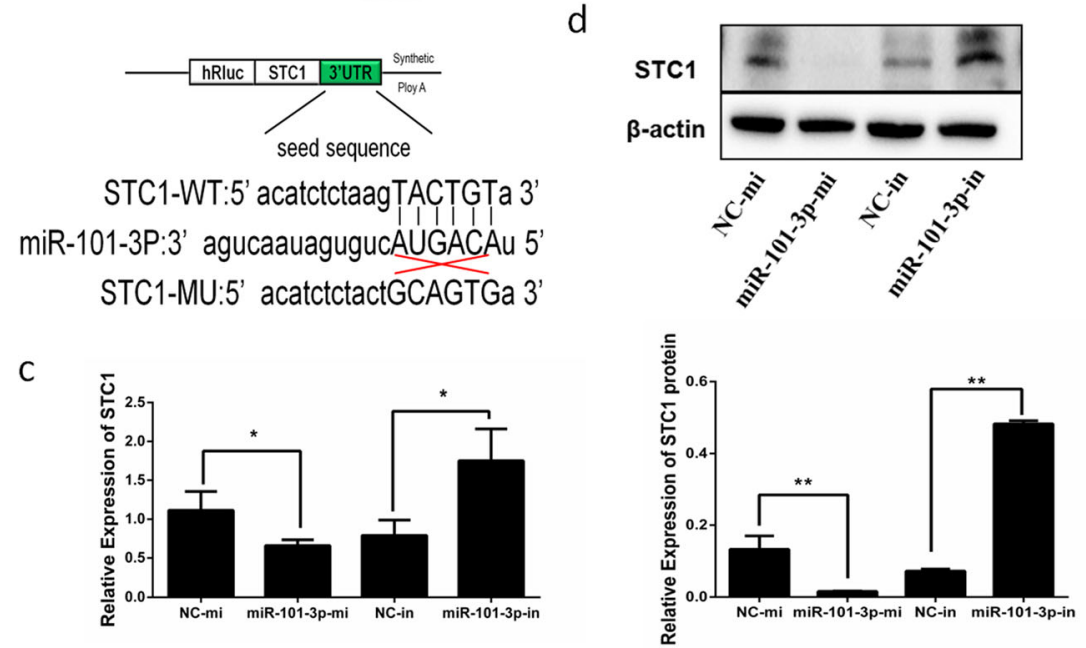

Fig. 2 MiR-101-3p specifically targets STC1 in goat granulosa cells. a Target sites for miR-101-3p in the STC1 3'-UTR and the construction of the luciferase expression vector (Luc) fused with the STC1 3'-UTR. wt represents the Luc reporter vector with the wild-type STC1 3'-UTR; mu represents the Luc reporter vector with the mutation at the miR-101-3p site in STC1 3'-UTR. b After HEK293T cells transfected miR-101-3p mimics (miR-101-3p-mi) or mimics NC (NC-mi), miR-101-3p inhibitors (miR-101-3p-in) or inhibitors NC (NC-in) with wt-/mu-Luc reporter vectors for $48 \mathrm{~h}$, the relative luciferase activities are measured. The (c) mRNA or (d) protein expressions of STC1 in granulosa cells transfected with miR-101-3p-mi/in or $\mathrm{NC}$-mi/-in for $24 \mathrm{~h}$ or $48 \mathrm{~h}$ are quantified using RT-qPCR or Western blot, respectively. $\beta$-actin is used as an internal control. Values are expressed as mean \pm SD of $n=3$. ${ }^{*} P<0.05,{ }^{* *} P<0.01$. NS means no significance

number of S-phage cells (Fig. 5c). STC1 also attenuated miR-101-3p-weakened effects on cell proliferation by increasing EdU positive cells (Fig. 5d). Silencing STC1 promoted cells to G0/G1 phase (88.15\%) and reduced cell numbers at $\mathrm{S}$ phase (3.47\%) compared to $82.90 \%$ and $8.94 \%$ cells in NC group (Fig. 5c). The number of granulosa cells was repressed at G0/G1 phase (72.57\%) but elevated at S phase (19.16\%) after STC1 overexpression compared with the percentages in control group $(77.49 \%$ and $14.40 \%)$ (Fig. 5c). Co-transfection group also showed a neutral result regarding cell cycle distribution, $84.97 \%$ cells at G0/G1 phase, $8.57 \%$ cells at $S$ phase and $6.46 \%$ cells at G2 phase compared to $93.01 \%, 3.36 \%$ and $3.63 \%$ cells in miR-101-3pmi group (Fig. 5d). Thus, we speculate that $S T C 1$ promotes granulosa cell proliferation and miR-101-3p can inhibit granulosa cell proliferation via STC1.

To further explore the regulatory mechanism of miR101-3p's effects on granulosa cell proliferation, we detected the expressions of cell proliferation-related genes (CDK4, CCND1, CCNE1 and PCNA). Figure 6a shows that miR-101-3p overexpression suppressed the mRNA levels of CDK4, CCND1 and CCNE1, whereas miR-101$3 \mathrm{p}$ inhibition facilitated the mRNA levels of CCNE1. Down-regulation of CDK4, CCND1, CCNE1 and PCNA protein expression was observed in miR-101-3p-mi group, whereas opposite results were found in miR-1013p-in group (Fig. 6b). Cells transfected with si-STC1 blocked the expression of CDK4 and CCND1 but showed slight effects on CCNE1 or PTEN (Fig. 6c). STC1 overexpression accelerated CKD4, CCND1 and PCNA but restrained CCNE1 protein levels (Fig. 6c). Co-expression of miR-101-3p with STC1 exhibited an adiaphorous effect on CCND1, CCNE1 and PCNA but not on CDK4 (Fig. 6d). These findings indicate that miR-101-3p is capable of inhibiting granulosa cell proliferation by effects on CDK4, CCND1, CCNE1 and PCNA proliferation-related genes via $S T C 1$.

MiR-101-3p promotes granulosa cell apoptosis via STC1 Flow cytometry showed that after $24 \mathrm{~h}$ transfection, the total apoptotic rates of granulosa cells increased in miR101-3p-mi group (Fig. 7a). Cells treated with miR-101- 


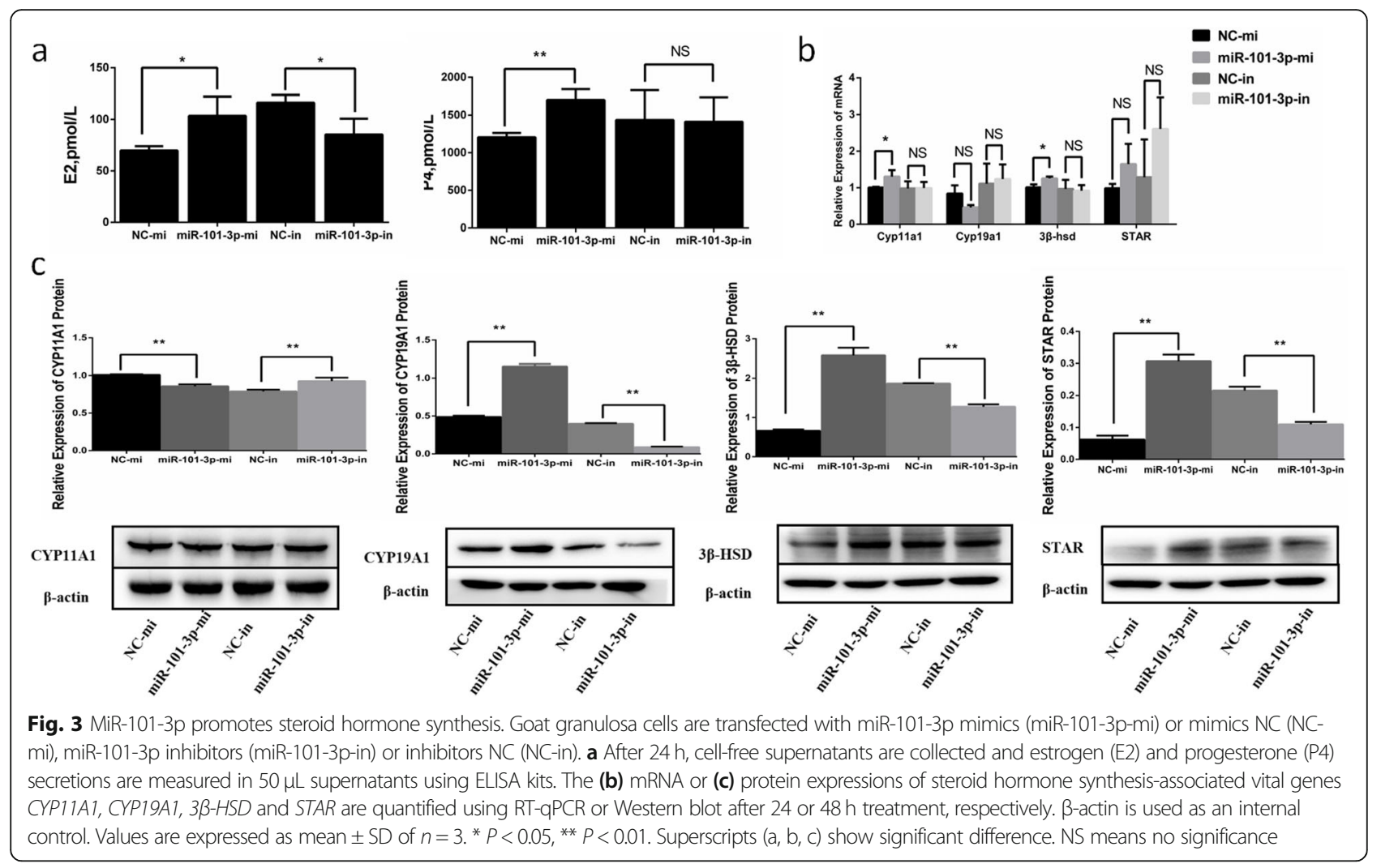

$3 p$-in took no effects on total apoptotic rates of granulosa cells, but exhibited decreased numbers of early-state apoptotic cells and increased numbers of late-state apoptotic cells (Fig. 7a). We detected the expressions of valid apoptotic genes Bcl-2, Bax and p53. MiR-101-3p elevation promoted Bax and inhibited Bcl-2 in mRNA and protein levels, and only promoted p53 protein levels (Fig. 8a, b). Cells with miR-101-3p inhibitors suppressed Bax and p53 and stimulated Bcl-2 in mRNA and protein levels (Fig. 8a, b). Furthermore, miR-101-3p decreased the ratio of $\mathrm{Bcl}-2 / \mathrm{Bax}$ (Fig. 8c), clarifying that miR-101$3 p$ promotes granulosa cell apoptosis. We also detected the influence of $S T C 1$ in granulosa cell apoptosis. Figure $7 \mathrm{~b}$ illustrates increased total apoptotic cells after cells transfected with si-STC1. Inducing STC1 resulted in decreased apoptotic cell numbers, and inhibited the positive effects of miR-101-3p on granulosa cell apoptosis (Fig. 7b, c). Western blot demonstrated that the protein expressions of Bax, p53 and Caspase 3 were improved, accompanied by restraining Bcl-2 in si-STC1 group, whereas STC1 overexpression showed opposite results (Fig. 8d). The ratio of Bcl-2/Bax was significantly increased by STC1 (Fig. 8f), implying that STC1 inhibits granulosa cell apoptosis. The effects of miR-101-3p on Bax, Bcl-2 and p53 were partially decreased after STC1 overexpression (Fig. 8e, f). Thereby it suggests that miR- 101-3p promotes granulosa cell apoptosis, at least in some degree, through STC1.

\section{MiR-101-3p inhibits PI3K-AKT-mTOR pathway via STC1}

PI3K-AKT-mTOR pathway plays a crucial function on cell growth, proliferation, apoptosis and other processes [32]. Therefore, we explored whether miR-101-3p and STC1 can affect the key genes in PI3K-AKT-mTOR pathway. MiR101-3p overexpression promoted PTEN, inhibited PI3K, AKT and mTOR protein levels, as well as triggered the activation of AKT and mTOR (Fig. 9a). MiR-101-3p inhibition significantly reduced PTEN expression and promoted PI3K and mTOR protein levels (Fig. 9a). Phosphorylation expressions of AKT and mTOR were also promoted between miR-101-3p-in and NC-in groups (Fig. 9a). These data support the passive role of miR-101-3p on PI3K-AKT-mTOR pathway. STC1 depletion decreased the protein levels of PI3K, p-AKT, mTOR and p-mTOR but increased those of PTEN and AKT (Fig. 9b). We observed increased PI3K, AKT, mTOR and p-mTOR expressions and decreased PTEN expression after $S T C 1$ was induced (Fig. 9b). The effects of miR-101-3p on PI3K, PTEN, AKT, p-AKT, mTOR and p-mTOR were partially neutralized after STC1 overexpression (Fig. 9c), suggesting that miR-101-3p inhibits PI3K-AKT-mTOR pathway via STC1. 


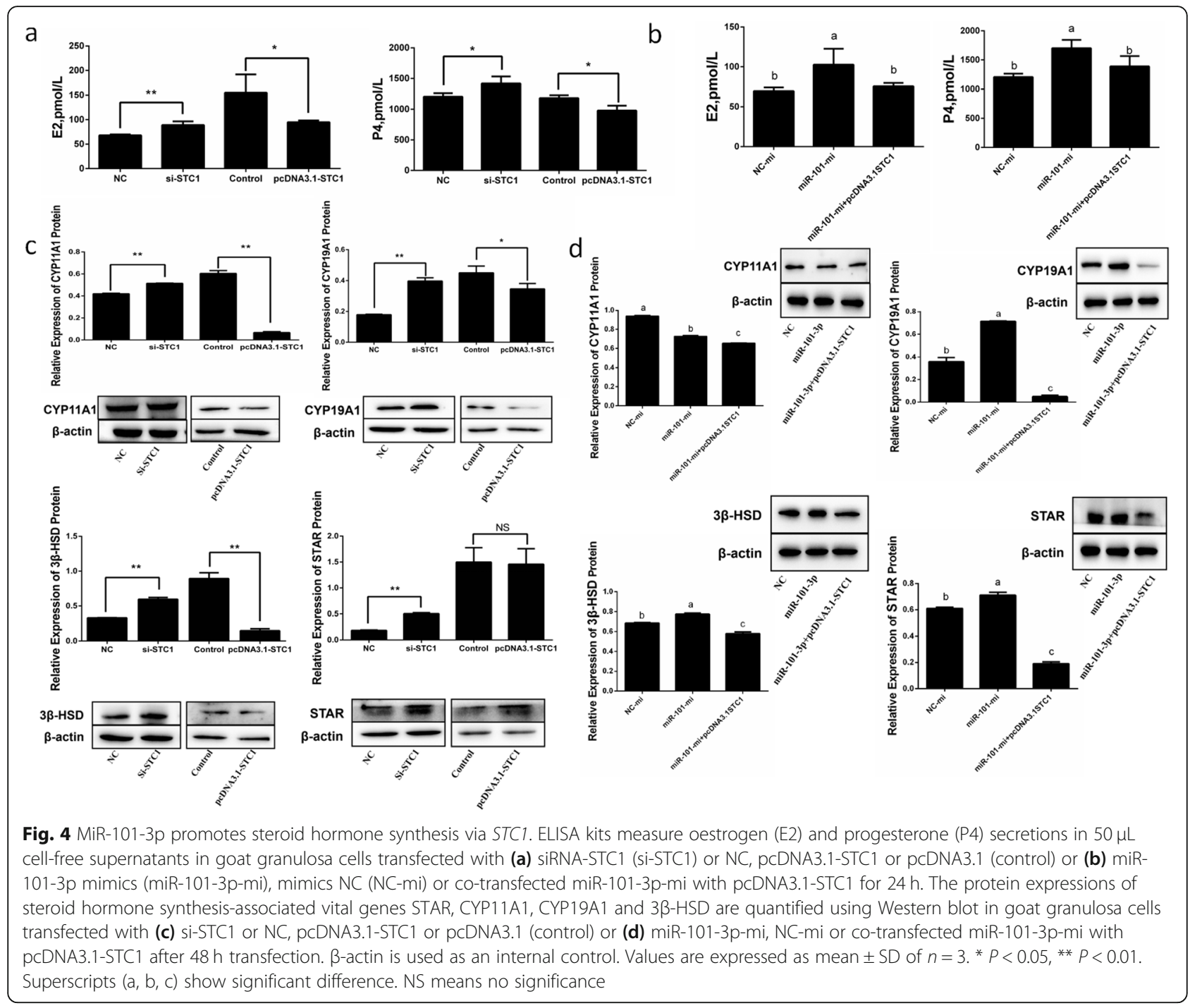

Effects of miR-101-3p and STC1 on ovarian development in mice

Then we confirmed whether miR-101-3p functions on mammalian ovarian physiological activities in vivo. The distribution and expression levels of miR-101-3p in mouse ovaries were identified through FISH. In NC group, miR101-3p expressed in granulosa cells of primary, secondary follicles, theca cells, stroma cells, and corpus luteum partially (Fig. 10a). MiR-101-3p expressed in most regions of the ovary in miR-101-3p-ag group, incorporating theca cells, granulosa cells of primordial, primary, secondary, mature and growing follicles, corpus luteum and stroma cells (Fig. 10a). MiR-101-3p expression in the ovary was marginal, and only a small amount of fluorescence was observed in the local stroma cells in miR-101-3p-antag group (Fig. 10a). Immunohistochemistry exhibited that STC1 expressed in theca cells and granulosa cells of follicles widely. MiR-101-3p overexpression and STC1 depletion suppressed STC1 expression in the ovaries, whereas
miR-101-3p-antag treatment promoted STC1 expression (Fig. 10b, Table 2). We then observed the morphology and counted follicles numbers at each stage after treated mice ovaries were stained with HE. Small and stunted ovarian fragments were observed in miR-101-3p-ag and si-STC1 groups, while miR-101-3p inhibition exhibited an opposite result (Fig. 10c). The results showed decreased numbers of primary, secondary, early antral, antral and total follicles in miR-101-3p-ag group, decreased numbers of secondary, antral and total follicles in si-STC1 groups (Fig. 10c, d). Increased numbers of primary and early antral follicles and decreased numbers of secondary and antral follicles were exhibited in miR-101-3p-antag group compared with NC (Fig. 10c, d).

\section{Discussion}

Ovarian activity is the key to a successful reproduction [1]. The complex changes in tissue components and functions require a high degree of spatiotemporal 


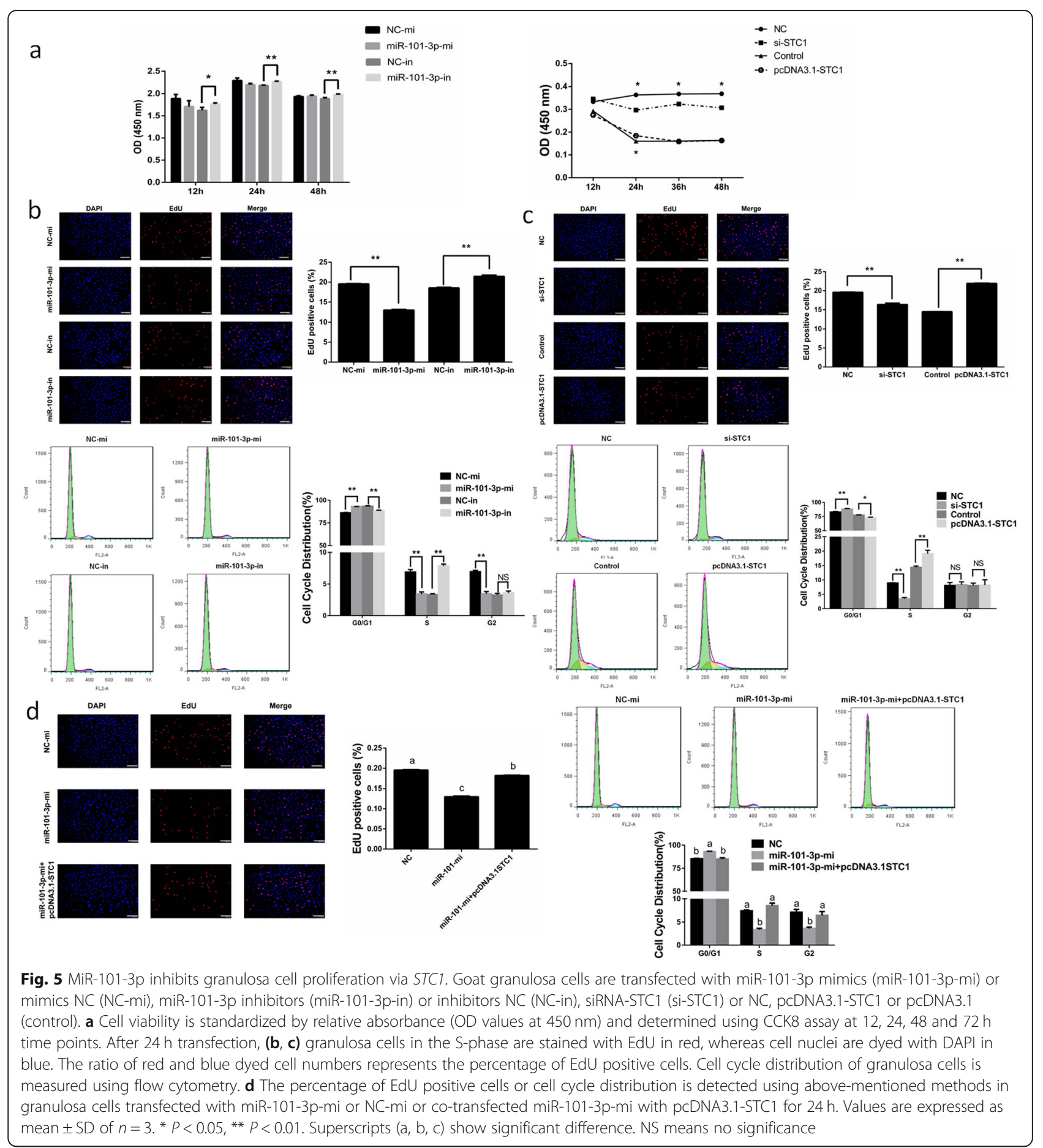

synergy in proliferation, apoptosis and differentiation of diverse cells in follicle, corpus luteum and ovarian stroma [2]. Studies indicate that miRNAs take crucial effects on ovarian activities by regulating genes related to ovarian development [7-15]. Our previous work shows that miR-101-3p expresses differentially in dairy goat ovaries compared single with multiple litter sizes, indicating it may influence ovarian development (not published). Hence, more investigations are needed to recognize the specific functions of miR-101-3p on goat ovaries.

Firstly, our present work chose goat granulosa cells to study the potential molecular functions of miR-101-3p, since granulosa cells of ovarian follicles participate in oocyte nourishing, secreting steroid hormones that regulate ovarian function $[2,3]$. Herein, a complete 


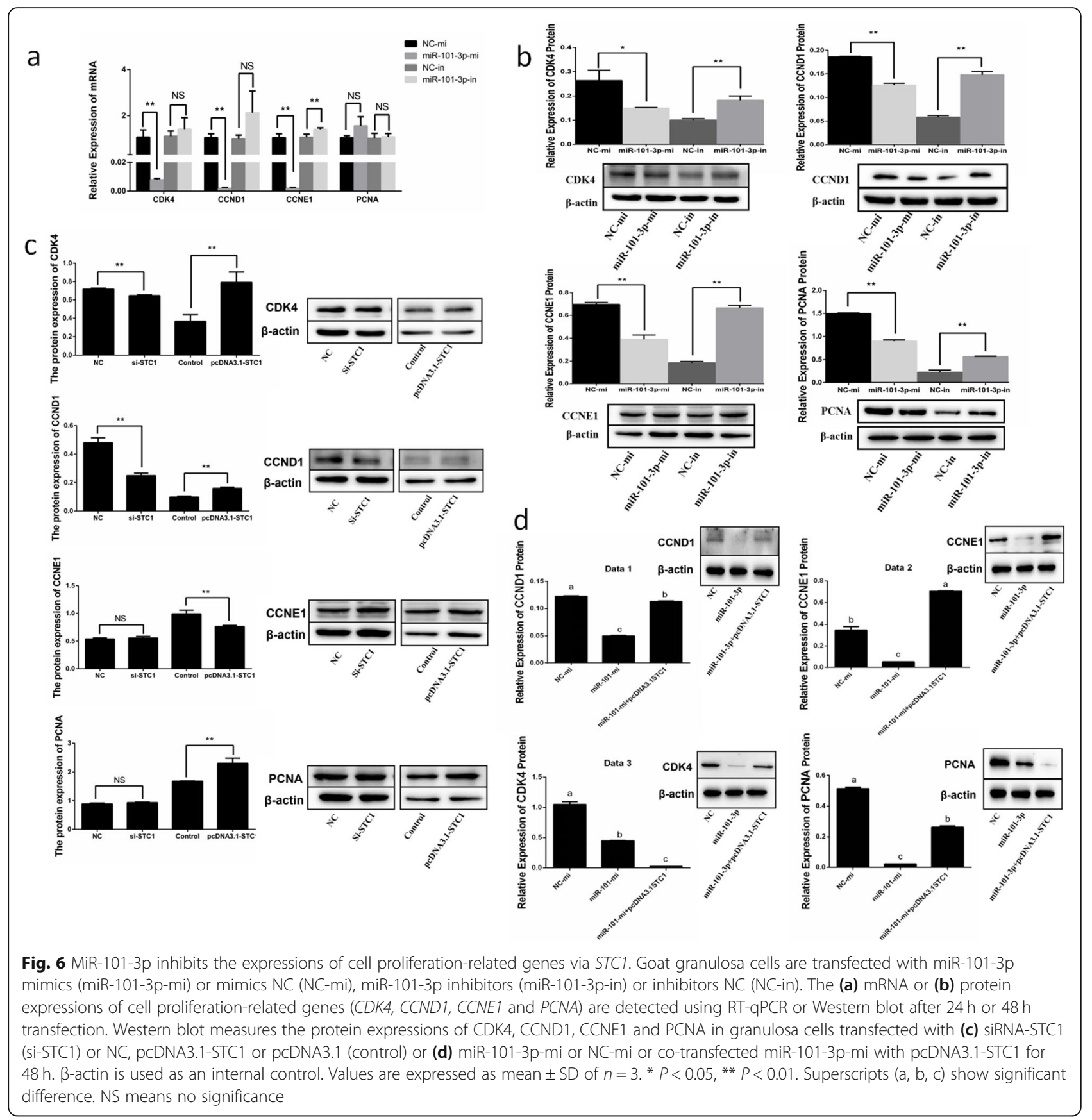

transcriptome dataset detailing the differentially expressed unigenes after miR-101-3p overexpression in granulosa cells was accomplished using RNA-Seq approach. A total of 142 DEGs was identified compared miR-101-3p mimics with NC groups, with 78 downregulated genes including C4BPA, STC1, EDN2, KCP and $P D Z K 1$ and 64 up-regulated genes including $F S H B$, $B M P E R, C N N M 1, N F I B$ and NR4A3. C4BPA suppresses complement activation by binding to $C 4 \mathrm{~B}$ and regulates lipid metabolism, inflammation and coagulation pathways [33]. STC1 is recently reported to participate in a variety of reproductive-related processes, including ovarian growth and development [22-26]. EDN2 is proposed to accelerate ovulation as a granulosa cell-derived contractile signal [34]. KCP interacts with BMPs and the BMP type I receptor, thus facilitates BMP signaling in a paracrine manner [35]. PDZK1 interacts with vital membrane-associated proteins and transporters and is identified as an estrogen-regulated gene [36]. $F S H B$, as one of the follicle stimulating hormone (FSH) subunits, regulates crucial reproductive functions such as steroid production and ovarian development via FSH signaling 


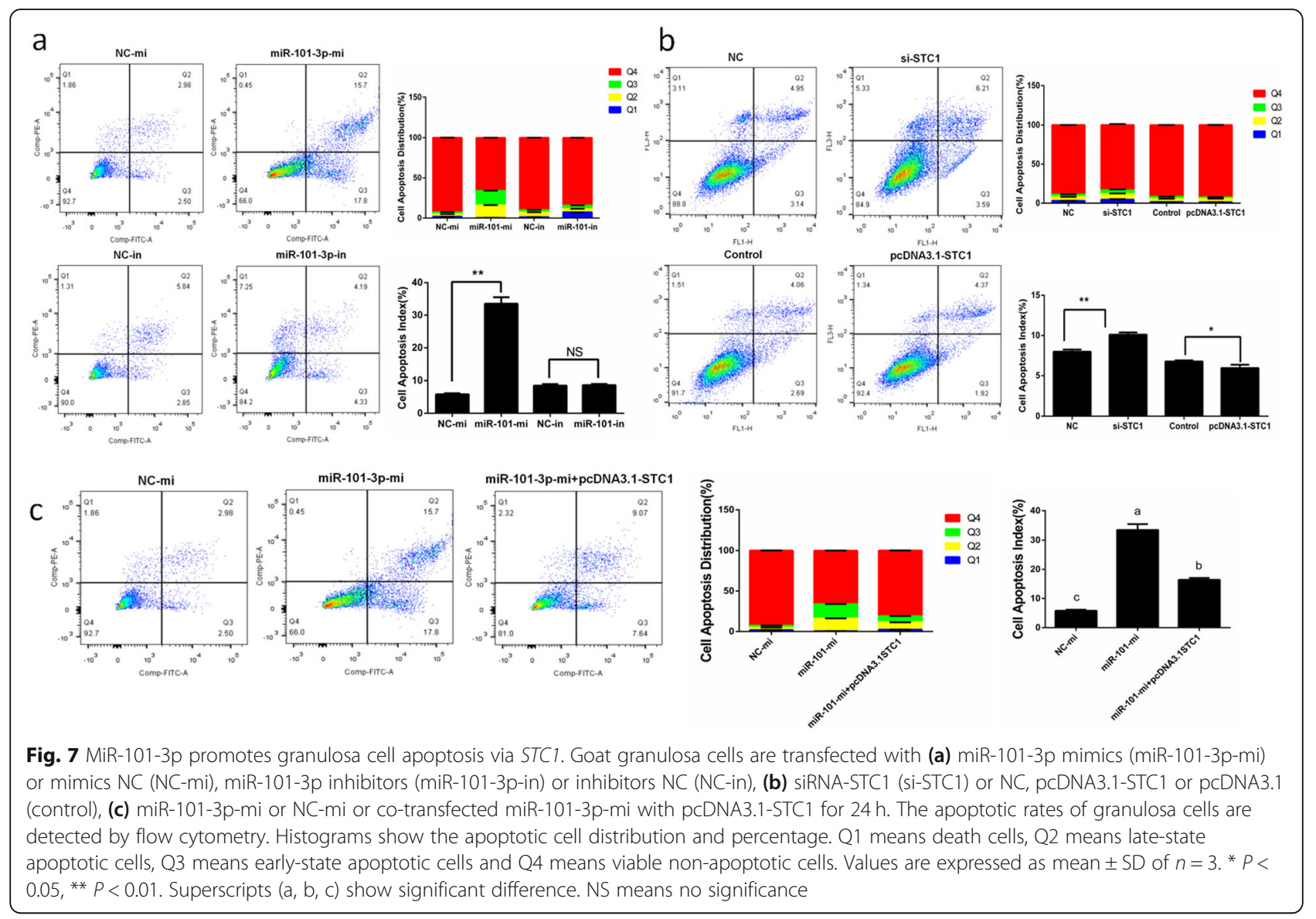

[37]. BMPER is a BMP-binding endothelial cell precursor-derived regulator and involved in various cell biology by BMP signaling [38]. CNNM1 works in selfrenewal of stem cells of spermatogonia and cell cycle regulation [39]. $N F I B$ belongs to transcription factors, implicated in cell differentiation, growth and other processes [40]. NR4A3 regulates the transcription of overlapping target genes implicated in a series of cellular processes and works as a nuclear receptor [41]. This study also expanded the amount of genetic information available and provided a profile of physiological processes of these DEGs. Neuroactive ligand-receptor interaction, natural killer cell mediated cytotoxicity, cytokine-cytokine receptor interaction and complement and coagulation cascades were among the significantly enriched pathways. Our results furnish a first step toward a modified understanding of the functions of miR-101-3p on goat granulosa cells.

Next, we specialized in the specific effects of miR-101$3 \mathrm{p}$ on goat granulosa cells in vitro. From detected DEGs, we selected STC1 for further research for the following reasons: (1) there is a miR-101-3p binding site of STC13'-UTR, (2) STC1 is involved in the most significant down-regulated DEGs and (3) STC1 is considered as a vital ovarian regulator. STC1 shows a gestational and nursing state function, identifies cholesterol or lipid storage droplets which were subcellular luteal cell targets in ovaries [23, 24]. STC1 also inhibits FSH-, LH- and hCGstimulated progesterone synthesis in rat granulosa cells and bovine luteal cells $[25,26]$. In swine ovarian follicles, STC1 acts as a physiological regulator of follicular function and modulates redox status in granulosa cells [42]. Our work showed that miR-101-3p targeted the 3'-UTR of STC1 and further blocked its mRNA and protein levels, revealing that miR-101-3p specifically targets STC1. Different steroid hormones affect follicular development via granulosa cell growth and follicular fluid formation, including cell proliferation, apoptosis and angiogenesis within the follicle. E2 and P4 are well-known steroid hormones and regulate the expression of related genes involved in ovulation and luteal formation [31]. Thus this study detected the effects of miR-101-3p and STC1 on E2 and P4 and showed that miR-101-3p promoted and STC1 suppressed E2 and P4 secretions. We also found that miR101-3p increased CYP11A1 and 3 $\beta-H S D$ mRNA levels and STAR, CYP19A1 and 3 3 -HSD protein levels but decreased CYP11A1 protein levels. STC1 promoted the protein expressions of STAR, CYP11A1, CYP19A1 and 3 $\beta$ HSD. Thus we speculate that miR-101-3p and STC1 affect 


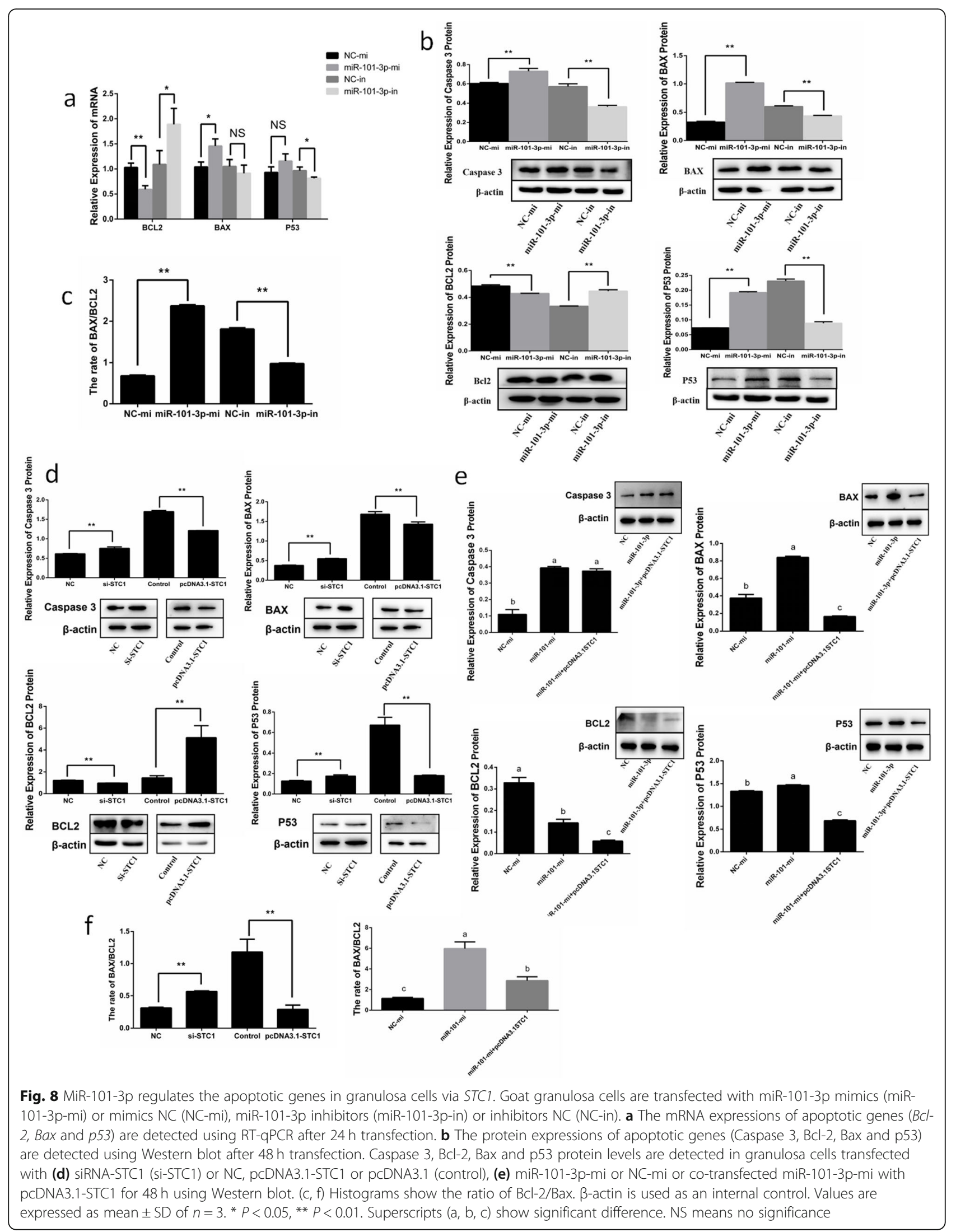


a

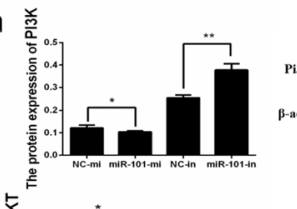

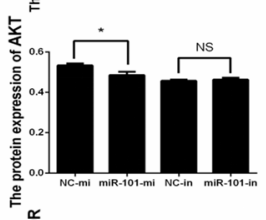

党

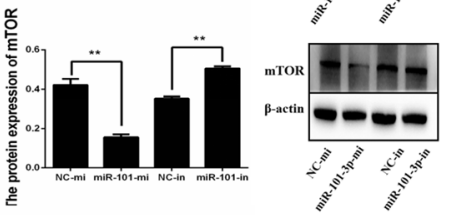

b

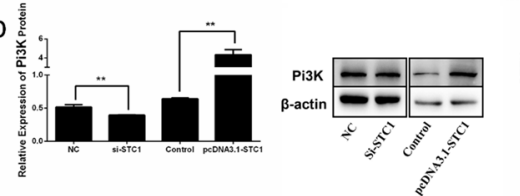

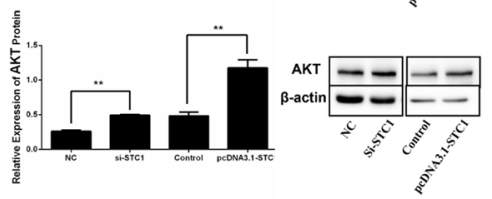

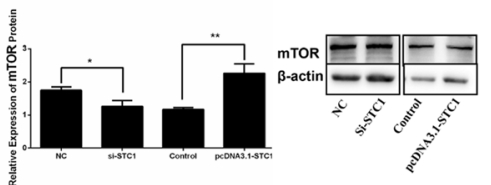

$\mathrm{C}$

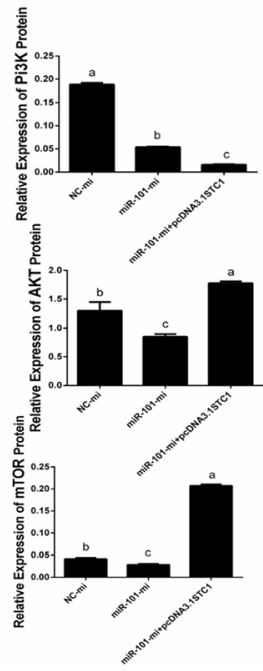

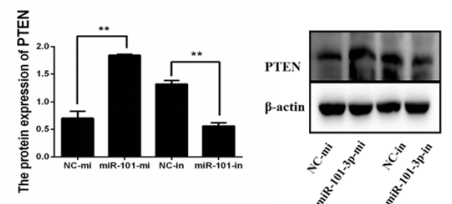
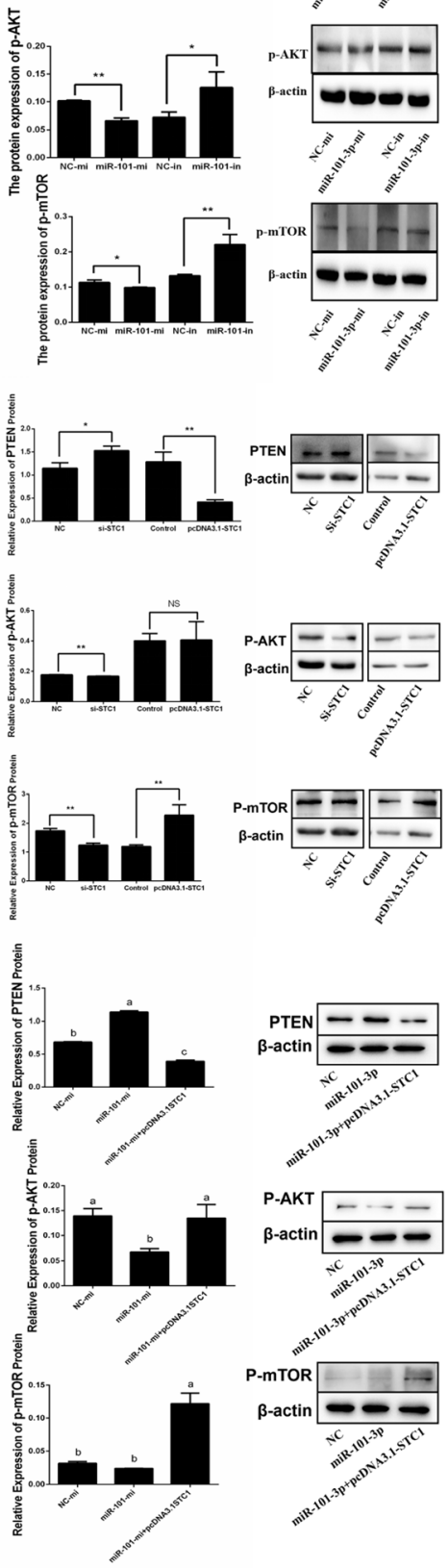

Fig. 9 MiR-101-3p inhibits PI3K-AKT-mTOR pathway via STC1. Goat granulosa cells are transfected (a) miR-101-3p mimics (miR-101-3p-mi) or mimics NC (NC-mi), miR-101-3p inhibitors (miR-101-3p-in) or inhibitors NC (NC-in), (b) siRNA-STC1 (si-STC1) or NC, pcDNA3.1-STC1 or pCDNA3.1 (control), (c) miR-101-3p-mi or NC-mi or co-transfected miR-101-3p-mi with pcDNA3.1-STC1 for $48 \mathrm{~h}$. Cytosolic protein and related phosphorylation levels for PI3K, AKT, mTOR and PTEN are analysed using Western blot. $\beta$-actin is used as an internal control. Values are expressed as mean \pm SD of $n=3$. ${ }^{*} P<0.05,{ }^{*} P<0.01$. Superscripts $(a, b, c)$ show significant difference. NS means no significance 


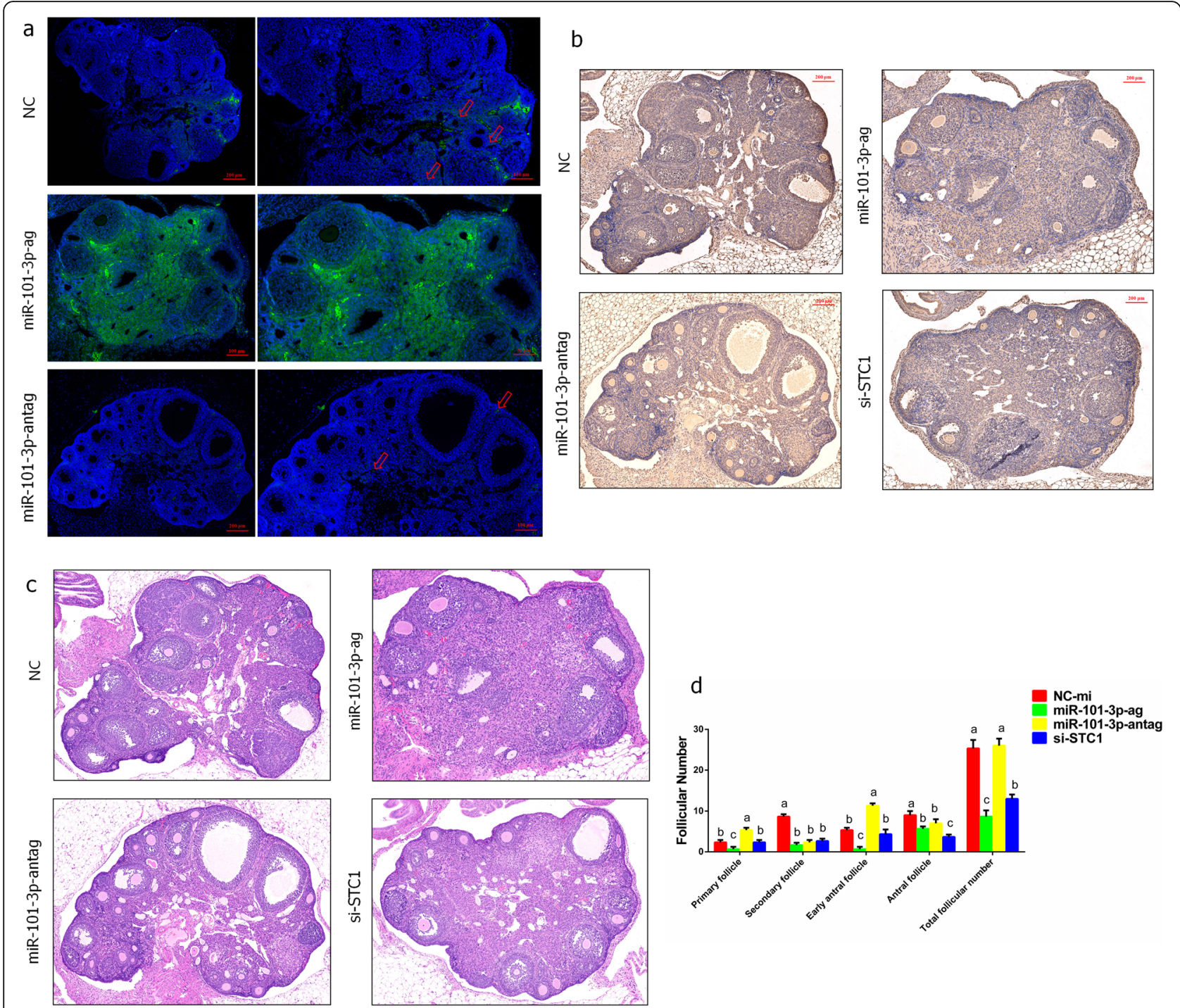

Fig. 10 Effects of miR-101-3p and STC1 on ovarian development in mice. Twelve mice are divided into four groups and treated with NC (100 $\mu \mathrm{L}$ saline), miR-101-3p agonists (miR-101-3p-ag; $10 \mathrm{nmol}$ per time, with $100 \mu \mathrm{L}$ saline), miR-101-3p antagonist (miR-101-3p-antag; $20 \mathrm{nmol}$ per time, with $100 \mu \mathrm{L}$ saline) or siRNA-STC1 (si-STC1; $20 \mathrm{nmol}$ per time, with $100 \mu \mathrm{L}$ saline). a FISH is performed to identify the distribution and expression levels of miR-101-3p in mouse ovaries. The nucleus stained by DAPI is blue under ultraviolet excitation, and the positive expression is green fluorescence of the corresponding fluorescein-labelled FAM. The red arrows represent a small amount of fluorescence of miR-101-3p. $\mathbf{b}$ Immunohistochemistry exhibits the expressions of STC1 in dissected mouse ovaries. SABC-positive cells are dyed in brown, while cell nuclei are dyed with DAPI in blue. The area of positive cells (Area), mean optical density (Mean Density), and integrated optical density (IOD) are effective indicators for semi-quantitative analysis of immunohistochemistry results. c The mouse ovarian morphology is observed using HE staining. The sections are stained with hematoxylin in blue purple, and stained with eosin in red. $\mathbf{d}$ After HE staining, the follicles are counted at each stage. Follicle counting principles are as follows: primordial follicles: oocytes surrounded by a layer of flat granulosa cells or mixed cells of flat and cubic granulosa cells (total cell number < 7); primary follicles: oocytes surrounded by more than 7 cubic granulosa cells; secondary follicles: oocytes surrounded by more than 2 layers granular cells; early antral follicles: oocytes surrounded by 2-4 layers granulosa cells and contained a follicular antrum (diameter $<20 \mu \mathrm{m}$ ); antral follicles: follicles with an obvious follicular antrum. Red lines represent scale bars. Values are expressed as mean \pm SD of $n=3$. Superscripts $(a, b, c)$ show significant difference

E2 and P4 secretions via STAR, CYP11A1, CYP19A1 and $3 \beta$-HSD steroid hormone synthesis-associated genes. Coexpression of miR-101-3p with STC1 exhibited an adiaphorous effect on E2 and P4 secretions and CYP11A1, CYP19A1, 3 $\beta$-HSD and STAR expressions. Therefore, the regulation of miR-101-3p on steroid hormone synthesis in granulosa cells partly relies on STC1.

The proliferation and apoptosis of granulosa cells are closely related to ovarian development $[2,3]$. Accordingly, we explored the mechanism of miR-101-3p and 
Table 2 Identification of STC1 expression in mouse ovary by immunohistochemical

\begin{tabular}{llllll}
\hline Index & & STC1 & & \\
\cline { 3 - 6 } & & NC & miR-101-3p-ag & miR-101-3p-antag & si-STC1 \\
\hline Area & Mean & 410.69 & 224.44 & 290.36 & 304,008 \\
Mean deneity & SUM & 350,725 & 244,639 & 0.17 & 252,695 \\
& Mean & 0.12 & 0.12 & 175.31 & 0.10 \\
IOD & SUM & 105.10 & 130.78 & 53.71 & 143.69 \\
& Mean & 58.06 & 30.56 & $56,240.16$ & 20.47 \\
IOD/Area & SUM & $49,585.84$ & $33,311.77$ & $0.1849^{* *}$ & $0.1154^{*}$ \\
\hline
\end{tabular}

Area: the area of positive cells, Mean density: mean optical density, IOD: integrated optical density. ${ }^{*} P<0.05,{ }^{*} P<0.01, n=3$ independent

experiments (mean $\pm \mathrm{SD}$ )

STC1 in cellular survival capabilities of goat granulosa cells. The study revealed that miR-1013p inhibited, while STC1 promoted granulosa cell proliferation. Coexpression of miR-101-3p with STC1 indicated a neutral effect. Our data also showed that miR-101-3p inhibited CDK4, CCND1, CCNE1 and PCNA expressions while STC1 inhibition blocked CDK4 and CCND1 protein expressions. STC1 overexpression accelerated CKD4, CCND1 and PCNA but restrained CCNE1 protein levels, and mitigated the miR-101-3p's role on CCND1, CCNE1 and PCNA. These findings indicate that miR-101-3p inhibits granulosa cell proliferation by regulating $C D K 4$, $C C N D 1, C C N E 1$ and $P C N A$ proliferation-related genes via STC1. FCM assay demonstrated that miR-101-3p promoted and STC1 inhibited the apoptotic rates of granulosa cells. MiR-101-3p induced pro-apoptotic Bax and p53 expressions and reduced anti-apoptotic Bcl-2 expression. STC1 decreased the expressions of Bax, p53 and proapoptotic Caspase3 and restrained that of Bcl-2. Moreover, STC1 partly alleviated the effects of miR-101-3p on granulosa cell apoptotic rates and Bcl-2, Bax, p53 expressions. Thus, we speculate that miR-101-3p promotes granulosa cell apoptosis by regulating $B c l-2, B a x, p 53$ and caspase 3 via STC1 depletion.

PI3K-AKT-mTOR signalling pathway has an imperative role on protein synthesis and cellular processes including proliferation and apoptosis through co-regulated proteins [32]. PI3K is a key upstream motivator of $A K T$. $A K T$ can give rise to $m T O R$ phosphorylation, which also mediates metabolism processes to maintain cell growth and proliferation [43]. The tumour-suppressor PTEN is a vital passive mediator of cell-survival signalling pathways initiated by PI3K [44]. It's also reported the impacts of miR-101-3p on PI3K-AKT pathway in various kind of cells. In Saos-2 cells, miR-101 transfection inhibits the mRNA and protein expressions of mTOR, which consequently improves cell apoptosis and suppresses cell proliferation [16]. MiR-101 overexpression disrupts the PI3K-AKT pathway and promotes $\mathrm{Bcl} 2$-regulated apoptosis induced by RLIP76 in prostate cancer cells [17]. MiR-101 represses tumour growth and migration by down-regulating ROCK1 and inactivating PI3K-AKT and JAK-STAT pathways in osteosarcoma cells [18]. According to these reports, we explored whether miR-101-3p and STC1 could affect PI3K-AKT-mTOR pathway in goat granulosa cells. Our results showed that miR-101-3p inhibited PI3K, AKT and mTOR but enhanced PTEN protein levels. MiR-101-3p also triggered the activation of AKT and mTOR. We observed increasing PI3K, AKT, mTOR and p-mTOR expressions and decreasing PTEN expression after STC1 was induced. The effects of miR-101-3p on motivation and expression of these key proteins were partially alleviated via STC1. These findings support that miR-101-3p can regulate granulosa cell proliferation and apoptosis through STC1 by inhibiting the PI3K-AKT-mTOR pathway.

The above experiments show that miR-101-3p can regulate biological processes of goat granulosa cells cultured in vitro through the target gene STC1. However, whether miR-101-3p affects ovarian physiological activities consistently in vivo requires further research. Therefore, mouse ovaries were used to study miR-101$3 p$ functions. Mice were randomly divided into four groups and were injected with intraperitoneal drugs. FISH results showed that miR-101-3p expressed or marginal expressed in most regions of the ovaries in miR101-3p-ag or -antag groups. Hence, miR-101-3p agonists and antagonists were efficient and available for further research. We demonstrated that miR-101-3p overexpression and STC1 depletion inhibited, whereas miR-101-3p inhibition promoted $S T C 1$ expression in mouse ovaries, indicating that miR-101-3p inhibits STC1 in mouse ovaries. Moreover, miR-101-3p exhibited unusual ovarian development functions, as reflected by small and stunted ovarian fragments and decreased numbers of various follicles. We also observed consistent results after STC1 was inhibited. Thus we speculate that miR101-3p disrupts ovarian development in mouse ovaries and may partly performs its effects by STC1. The in vivo experiment results showed that the developmental status 
of mouse follicles coincided with miR-101-3p promoting apoptosis and inhibiting proliferation of granulosa cells in vitro, indicating that miR-101-3p may regulate ovarian development in dairy goats. Although, big species differences are present between dairy goat and mouse, miRBase database (http://www.mirbase.org/) shows that the seed sequences of chi-miR-101-3p and mmu-miR-101$3 p$ are similar (Fig. S3). The seed sequences (2-8 nts) which located at $5^{\prime}$ end of miRNAs, can pair with the $3^{\prime}$-UTR of their target genes and then trigger mRNA or/ and protein degradation. The miRNA binding sites are conserved in multiple species $[4,5]$. Hence, the highly conserved structure and function of miRNAs demonstrate the feasibility of our study using acknowledged model animals in vivo.

\section{Conclusion}

In conclusion, 78 down-regulated and 64 up-regulated DEGs were identified after miR-101-3p overexpression in goat granulosa cells using RNA-Seq. GO terms and KEGG pathway analysis demonstrated that DEGs could participate in the regulation of ovarian growth and development. In vitro, miR-101-3p targeted STC1, one of down-regulated DEGs directly and inhibited its expressions in goat granulosa cells. MiR-101-3p induced E2 and P4 secretions by STAR, CYP19A1, CYP11A1 and $3 \beta-$ $H S D$ steroid hormone synthesis-associated genes partially via STC1 depletion. MiR-101-3p also inhibited the proliferation and promoted the apoptosis of granulosa cells by regulating PI3K-AKT pathway key genes PI3K, PTEN, AKT and $m T O R$ via STC1 depletion. In vivo, miR-101-3p inhibited STC1 expression and ovarian development, while STC1 promoted ovarian development in mouse ovaries. Our results provided a theoretical basis and experimental evidence for miR-101-3p functions on goat ovarian development.

\section{Supplementary information}

Supplementary information accompanies this paper at https://doi.org/10. 1186/s40104-020-00506-6.

Additional file 1: Figure S1. The transfection efficiency of miR-101-3p and STC1.

Additional file 2: Figure S2. High-throughput sequencing of RNA

Additional file 3: Figure S3. The seed sequences of miR-101-3p in goat and mouse.

Additional file 4: Table S1. The sequences of miR-101-3p mimics, 101$3 p$ inhibitors, NC, inhibitor NC and si-STC1.

Additional file 5: Table S2. The validated primers used for RT-PCR.

Additional file 6: Table S3. Distribution of effective sequences in reference genomes.

Additional file 7: Table S4. The up- and down-regulated genes in the miR-101-3p mimics VS negetive control (NC) groups.

Additional file 8: Table S5. GO enrich of differentially expressed genes.
Additional file 9: Table S6. Pathway annotations of differentially expressed genes.

\section{Abbreviations}

DEGs: Differentially expressed unigenes; E2: Estrogen; P4: Progesterone; FISH: Fluorescence in situ hybridisation; HE: Hematoxylin-eosin; nts: Nucleotides; UTR: Untranslated region; STC: Stanniocalcin; RNA-Seq: Highthroughput sequencing of RNA; FBS: Foetal bovine serum; RPKM: Reads per kilobase transcriptome per million mapped reads; GO: Gene Ontology; RTqPCR: Quantitative real-time PCR; ELISA: Enzyme-linked immunosorbent assay; FCM: Flow cytometry assay; PI: Propidium iodide; CAMs: Cell adhesion molecules; CDS: Coding sequence; FSH: Follicle stimulating hormone

\section{Acknowledgements}

Not applicable.

\section{Authors' contributions}

The work was mainly conceived and designed by XPA, YYB and BYC. Experiments were performed by XPA and HDM. Experimental data were collected and analyzed by HDM and YHL. The manuscript was mainly written by $\mathrm{YHL}$ and revised by FL, GL and YXS. All the authors contributed to, read and approved the final manuscript.

\section{Funding}

This study was supported by the National Natural Science Foundation of China (31601925), Shaanxi Science and Technology Innovation Project Plan (2020ZDLNY02-01, 2020ZDLNY02-02, 2018ZDCXL-NY-01-04, 2018ZDCXL-NY01-02 and 2017ZDXM-NY-081) and Natural Science Foundation of Shaanxi Province (2020JQ-868)

Availability of data and materials

The datasets used and/or analyzed during the current study are available from the corresponding author on request.

\section{Ethics approval}

All animal care and procedures were performed in accordance with institutional and national guidelines and approved by the Institutional Animal Care and Use Committee of Northwest A\&F University (China).

Consent for publication

Not applicable.

\section{Competing interests}

The authors declare no competing financial interest.

\section{Author details}

${ }^{1}$ College of Animal Science and Technology, Northwest A\&F University, No. 22 Xinong Road, Yangling, Shaanxi 712100, P.R. China. ${ }^{2}$ College of Biological Science and Engineering, Shaanxi University and Technology, Hanzhong,

Shaanxi 723001, P.R. China. ${ }^{3}$ Henan Animal Health Supervision Institution, No. 91 Jingsan Road, Zhengzhou, Henan 450008, P.R. China.

Received: 3 April 2020 Accepted: 18 August 2020

Published online: 14 October 2020

\section{References}

1. Sheldon IM, Noakes DE, Dobson $\mathrm{H}$. The influence of ovarian activity and uterine involution determined by ultrasonography on subsequent reproductive performance of dairy cows. Theriogenology. 2000;54:409-19.

2. Macklon NS, Fauser BCJM. Aspects of ovarian follicle development throughout life. Horm Res. 1999;52:161-70.

3. Vanderhyden BC, Telfer EE, Eppig JJ. Mouse oocytes promote proliferation of granulosa cells from preantral and antral follicles in vitro. Biol Reprod. 1992:46:1196-204.

4. Smirnova L, Grafe A, Seiler A, Schumacher S, Wulczyn FG. Regulation of miRNA expression during neural cell specification. Eur J Neurosci. 2005;21: 1469-77.

5. Ambros V. MicroRNA pathways in flies and worms: growth, death, fat, stress, and timing. Cell. 2003;113:673-6. 
6. Carlo M, Croce MD. Causes and consequences of miRNA dysregulation in cancer. Nat Rev Genet. 2009;10:704-14.

7. Yao G, Yin M, Lian J, Tian H, Liu L, Li X, et al. MicroRNA-224 is involved in transforming growth factor- $\beta$-mediated mouse granulosa cell proliferation and granulosa cell function by targeting Smad4. Mol Endocrinol. 2010;24: 540-51.

8. Yin M, Lu M, Yao G, Tian H, Sun F. Transactivation of microRNA-383 by steroidogenic factor-1 promotes estradiol release from mouse ovarian granulosa cells by targeting RBMS1. Mol Endocrinol. 2012;26:1129-43.

9. Xu S, Katja LM, Yang BB, Wu D, Li J. Micro-RNA378 (miR-378) regulates ovarian estradiol production by targeting aromatase. Endocrinology. 2011; 152:3941-51.

10. Xu L, Sun H, Zhang M, Jiang Y, Zhang C, Zhou J, et al. MicroRNA-145 protects follicular granulosa cells against oxidative stress-induced apoptosis by targeting Kruppel-like factor 4. Mol Cell Endocrinol. 2017:5:138-47.

11. Yang $X$, Zhou Y, Peng S, Wu L, Lin HY, Wang S, et al. Differentially expressed plasma microRNAs in premature ovarian failure patients and the potential regulatory function of miR-23a in granulosa cell apoptosis. Reproduction. 2012;144:235-44

12. Lin F, Li R, Pan ZX, Zhou B, Yu DB, Wang XG, et al. miR-26b promotes granulosa cell apoptosis by targeting ATM during follicular atresia in porcine ovary. PLoS One. 2012;7:e38640.

13. Liu LZ, Li C, Chen Q, Jing Y, Carpenter R, Jiang Y, et al. MiR-21 induced angiogenesis through AKT and ERK activation and HIF-1a expression. PLoS One. 2011;6:e19139.

14. Donadeu FX, Schauer SN. Differential miRNA expression between equine ovulatory and anovulatory follicles. Domest Anim Endocrinol. 2013;45:122-5.

15. Mcbride D, Carre W, Sontakke SD, Hogg CO, Law A, Donadeu FX, et al. Identification of miRNAs associated with the follicular-luteal transition in the ruminant ovary. Reproduction. 2012;144:221-33.

16. Lin S, Shao NN, Fan L, Ma XC, Shao ZW. Effect of microRNA-101 on proliferation and apoptosis of human osteosarcoma cells by targeting mTOR. J Huazhong Univ Sci Technolog Med Sci. 2014;34:889-95.

17. Yang J, Song Q, Cai Y, Wang P, Wang M, Zhang D. RLIP76-dependent suppression of PI3K/AKT/BCl-2 pathway by miR-101 induces apoptosis in prostate cancer. Biochem Biophys Res Commun. 2015;463:900-6.

18. Jiang R, Zhang C, Liu G, Gu R, Wu H. MicroRNA-101 inhibits proliferation, migration and invasion in osteosarcoma cells by targeting ROCK1. Am J Cancer Res. 2017;7:88-97.

19. Butkus A, Roche PJ, Fernley RT, Haralambidis J, Penschow JD, Ryan GB, et al. Purification and cloning of a corpuscles of Stannius protein from Anguilla australis. Mol Cell Endocrinol. 1987;54:123-33.

20. Lu M, Wagner GF, Renfro JL. Stanniocalcin stimulates phosphate reabsorption by flounder renal proximal tubule in primary culture. Am J Phys. 1994;267:1356-62.

21. Chang CM, Reddel RR. Identification of a second stanniocalcin CDNA in mouse and human: Stanniocalcin 2. Mol Cell Endocrinol. 1998;141:95-9.

22. Liu G, Yang G, Chang B, Mercado-Uribe I, Huang M, Zheng J, et al. Stanniocalcin 1 and ovarian tumorigenesis. J Natl Cancer Inst. 2010;102:812-27.

23. Stasko SE, Wagner GF. Stanniocalcin gene expression during mouse urogenital development: a possible role in mesenchymal-epithelial signalling. Dev Dyn. 2001;220:49-59.

24. Mark P, DG E, Wagner GF. Regulation of luteal cell big stanniocalcin production and secretion. Endocrinology. 2004;145:4204-12.

25. Paciga M, Mccudden CR, Londos C, Dimattia GE, Wagner GF. Targeting of big stanniocalcin and its receptor to lipid storage droplets of ovarian steroidogenic cells. J Biol Chem. 2003;278:49549-54.

26. Luo CW, Kawamura K, Klein C, Hsueh AJW. Paracrine regulation of ovarian granulosa cell differentiation by stanniocalcin (STC) 1: mediation through specific STC1 receptors. Mol Endocrinol. 2004;18:2085-96.

27. Grabherr MG, Haas BJ, Yassour M, Levin JZ, Thompson DA, Amit I, et al. Fulllength transcriptome assembly from RNA-Seq data without a reference genome. Nat Biotechnol. 2011;29:644-52.

28. Evelyn C, Michele M, Daniel B, Vivian L, Emily D, John M, et al. The gene ontology annotation (GOA) database: sharing knowledge in uniprot with gene ontology. Nucleic Acids Res. 2004;32:262-6.

29. Minoru K, Susumu G. KEGG: Kyoto encyclopedia of genes and genomes. Nucleic Acids Res. 1999;27:29-34

30. Zhang XH, Zhang YN, Li HB, Hu CY, Wang N, Cao PP, et al. Overexpression of miR-125b, a novel regulator of innate immunity, in eosinophilic chronic rhinosinusitis with nasal polyps. Am J Respir Crit Care Med. 2012;185:140-51.
31. Louis TM, Hafs HD, Morrow DA. Intrauterine administration of prostaglandin F2 alpha in cows: progesterone, estrogen, $\mathrm{LH}$, estrus and ovulation. Am J Obstet Gynecol. 1974;130:876-86.

32. Brunet A, Datta SR, Greenberg ME. Transcription-dependent and -independent control of neuronal survival by the PI3K-Akt signaling pathway. Curr Opin Neurobiol. 2001;11:297-305.

33. Buil A, Trégouet DA, Souto JC, Saut N, Germain M, Rotival M, et al. C4BPB/ C4BPA is a new susceptibility locus for venous thrombosis with unknown protein S-independent mechanism: results from genome-wide association and gene expression analyses followed by case-control studies. Blood. 2010; 115:4644-50.

34. Bridges PJ, Jo M, Al Alem L, Na G, Su W, Gong MC, et al. Production and binding of endothelin-2 (EDN2) in the rat ovary: endothelin receptor subtype a (EDNRA)-mediated contraction. Reprod Fert Dev. 2010;22:780-7.

35. Lin J, Patel SR, Wang M, Dressler GR. The cysteine-rich domain protein KCP is a suppressor of transforming growth factor beta/activin signaling in renal epithelia. Mol Cell Biol. 2006;26:4577-85.

36. Ghosh MG, Thompson DA, Weigel RJ. PDZK1 and GREB1 are estrogenregulated genes expressed in hormone-responsive breast cancer. Cancer Res. 2000;60:6367-75.

37. Zhang ZW, Findlay JK, Carson RS, Herington AC, Burger HG. Transforming growth factor beta enhances basal and FSH-stimulated inhibin production by rat granulosa cells in vitro. Mol Cell Endocrinol. 1988;58:161-6.

38. Dyer L, Wu Y, Moser M, Patterson C. BMPER-induced BMP signaling promotes coronary artery remodeling. Dev Biol. 2014;386:385-94.

39. Chandran U, Indu S, Kumar AT, Devi A, Khan I, Srivastava D, et al. Expression of CNNM1 and its association with stemness, cell cycle, and differentiation in spermatogenic cells in mouse testis. Biol Reprod. 2016;95:1.

40. Piper M, Barry G, Harvey TJ, Mcleay R, Richards LJ. NFIB-mediated repression of the epigenetic factor Ezh2 regulates cortical development. J Neurosci. 2014;34:2921-30.

41. Mullican SE, Zhang S, Konopleva M, Ruvolo V, Conneely OM. Abrogation of nuclear receptors NR4A3 and NR4A1 leads to development of acute myeloid leukemia. Nat Med. 2007;13:730-5.

42. Laura B, Giuseppina B, Simona B, Grasselli F. Stanniocalcin 1 affects redox status of swine granulosa cells. Regul Pept. 2011;168:45-9.

43. Sarbassov DD. Phosphorylation and regulation of Akt/PKB by the rictormTOR complex. Science. 2005;307:1098-101.

44. Jiang BH, Liu LZ. PI3K/PTEN signaling in angiogenesis and tumorigenesis. Adv Cancer Res. 2009:102:19-65.

\section{Ready to submit your research? Choose BMC and benefit from:}

- fast, convenient online submission

- thorough peer review by experienced researchers in your field

- rapid publication on acceptance

- support for research data, including large and complex data types

- gold Open Access which fosters wider collaboration and increased citations

- maximum visibility for your research: over $100 \mathrm{M}$ website views per year

At $\mathrm{BMC}$, research is always in progress.

Learn more biomedcentral.com/submissions 